\title{
A Broadband Look at the Old and New ULXs of NGC 6946
}

\author{
Hannah P. Earnshaw $^{1}$ (D), Brian W. Grefenstette ${ }^{1}$ (D), Murray Brightman ${ }^{1}$, Dominic J. Walton ${ }^{2}$ (D), Didier Barret ${ }^{3}$ (D), Felix Fürst ${ }^{4}$ (iD), \\ Fiona A. Harrison ${ }^{1}$, Marianne Heida ${ }^{1}$ (D), Sean N. Pike ${ }^{1}$ (D), Daniel Stern ${ }^{5}$ (D) and Natalie A. Webb ${ }^{3}$ \\ ${ }^{1}$ Cahill Center for Astronomy and Astrophysics, California Institute of Technology, Pasadena, CA 91125, USA; hpearn@ caltech.edu \\ ${ }^{2}$ Institute of Astronomy, Madingley Road, Cambridge CB3 0HA, UK \\ ${ }^{3}$ IRAP, Université de Toulouse, CNRS, UPS, CNES, Toulouse, France \\ ${ }^{4}$ European Space Astronomy Centre (ESAC), Science Operations Department, E-28692, Villanueva de la Cañada, Madrid, Spain \\ 5 Jet Propulsion Laboratory, California Institute of Technology, 4800 Oak Grove Drive, Pasadena, CA 91109, USA \\ Received 2019 April 5; revised 2019 May 7; accepted 2019 May 8; published 2019 August 9
}

\begin{abstract}
Two recent observations of the nearby galaxy NGC 6946 with NuSTAR, one simultaneous with an XMM-Newton observation, provide an opportunity to examine its population of bright accreting sources from a broadband perspective. We study the three known ultraluminous X-ray sources (ULXs) in the galaxy, and find that ULX-1 and ULX-2 have very steep power-law spectra with $\Gamma=3.6_{-0.3}^{+0.4}$ in both cases. Their properties are consistent with being super-Eddington accreting sources with the majority of their hard emission obscured and down-scattered. ULX-3 (NGC 6946X-1) is significantly detected by both XMM-Newton and NuSTAR at $L_{\mathrm{X}}=(6.5 \pm 0.1) \times 10^{39} \mathrm{erg} \mathrm{s}^{-1}$, and has a power-law spectrum with $\Gamma=2.51 \pm 0.05$. We are unable to identify a high-energy break in its spectrum like that found in other ULXs, but the soft spectrum likely hinders our ability to detect one. We also characterize the new source, ULX-4, which is only detected in the joint $X M M$ Newton and NuSTAR observation, at $L_{\mathrm{X}}=(2.27 \pm 0.07) \times 10^{39} \mathrm{erg} \mathrm{s}^{-1}$, and is absent in a Chandra observation 10 days later. It has a very hard cutoff power-law spectrum with $\Gamma=0.7 \pm 0.1$ and $E_{\text {cut }}=11_{-4}^{+9} \mathrm{keV}$. We do not detect pulsations from ULX-4, but its transient nature can be explained either as a neutron star ULX briefly leaving the propeller regime or as a micro-tidal disruption event induced by a stellar-mass compact object.
\end{abstract}

Key words: accretion, accretion disks - stars: black holes - stars: neutron - X-rays: binaries - X-rays: general

\section{Introduction}

The study of ultraluminous X-ray sources (ULXs) offers insight into some of the most extreme accretion processes in the universe (for a recent review, see Kaaret et al. 2017). This is indicated by their high luminosities $\left(L_{\mathrm{X}}>10^{39} \mathrm{erg} \mathrm{s}^{-1}\right)$, which imply either the presence of an intermediate-mass black hole (IMBH; $10^{2}<M_{\mathrm{BH}}<10^{5} M_{\odot}$; Colbert \& Mushotzky 1999), or apparent super-Eddington accretion onto a stellar-mass compact object (e.g., Sutton et al. 2013b; Bachetti et al. 2014). In recent years, broadband X-ray observations using $X M M$ Newton and NuSTAR have shown the spectral and timing properties of ULXs to be distinct from those of sub-Eddington accretion states, with characteristic broadened-disk or twocomponent shapes in the XMM-Newton band, a spectral turnover at $\gtrsim 5 \mathrm{keV}$, and in the case of especially good NuSTAR data above $\sim 20 \mathrm{keV}$, an additional steep power-law excess at the highest energies (e.g., Stobbart et al. 2006; Gladstone et al. 2009; Bachetti et al. 2013; Walton et al. 2014, 2015b; Mukherjee et al. 2015). These properties are consistent with a supercritical accretion regime onto a stellarmass compact object, in which a cooler component is emitted from an outer supercritical accretion disk launching an outflowing wind, and a hotter component is emitted from the inner accretion flow (e.g., Middleton et al. 2015a). Which component is dominant in the spectrum depends on the inclination and/or mass accretion rate in this model, and at very high inclinations/accretion rates the high-energy emission may be completely obscured and reprocessed, leaving an ultraluminous supersoft source with a spectrum entirely dominated by a soft thermal component (e.g., Di Stefano et al. 2004; Urquhart \& Soria 2016; Earnshaw \& Roberts 2017). Conversely, ULXs with spectra dominated by hard emission are more likely to be sources observed at low inclinations in this picture, and make particularly good targets for NuSTAR given its sensitivity to energies $>10 \mathrm{keV}$.

It was initially assumed that, due to their extreme luminosities, these stellar-mass objects would nonetheless be as massive as reasonably possible, and therefore be black holes (BHs) by default. However, the detection of pulsations and cyclotron absorption features from a number of ULXs has shown at least some fraction of ULXs to be neutron stars (NSs) instead, with luminosities 100-1000 times their isotropic Eddington limit (e.g., Bachetti et al. 2014; Fürst et al. 2016; Israel et al. 2017a, 2017b; Brightman et al. 2018; Carpano et al. 2018; Rodríguez Castillo et al. 2019; Sathyaprakash et al. 2019). Those ULXs confirmed to be NSs share the property of a hard spectrum that can be modeled as being dominated by a cutoff power-law emission component originating from the accretion column and providing the pulsed portion of the spectrum (Brightman et al. 2016; Walton et al. 2018a). While pulsations have not been detected from all ULXs, all of the highest quality spectra of super-Eddington-type ULXs are potentially consistent with various different NS accretion models (Koliopanos et al. 2017; Pintore et al. 2017; Walton et al. 2018b).

Most ULXs are persistently bright sources, some of which exhibit high levels of long-term, inter-observation variability (e.g., Fridriksson et al. 2008; Sutton et al. 2012; Grisé et al. 2013). This can sometimes be sufficient to cross the $L_{\mathrm{X}} \geqslant 10^{39} \mathrm{erg} \mathrm{s}^{-1}$ boundary, which is the empirical definition of a ULX, so that a source can appear as a ULX in some observations and not others (e.g., Lin et al. 2013; Earnshaw \& Roberts 2017). More dramatically, a small handful of ULXs will sometimes drop in flux by orders of magnitude or so much 
as to become undetectable (e.g., Walton et al. 2015a; Earnshaw et al. 2018). This latter scenario is observed in several of the ULX pulsars discovered to date, giving them an approximately bimodal light curve (Fürst et al. 2016; Tsygankov et al. 2016; Israel et al. 2017b). This can be explained by the "propeller effect," in which accretion is stopped during periods when the magnetospheric radius of the NS exceeds the corotation radius of the accretion disk (Illarionov \& Sunyaev 1975; Stella et al. 1986). ULXs have also been known to appear (e.g., Soria et al. 2012; Pintore et al. 2018) or disappear (often the case for classical outbursts that briefly exceed $10^{39} \mathrm{erg} \mathrm{s}^{-1}$ before returning to a quiescent state; e.g., Middleton et al. 2013).

NGC 6946 is a nearby spiral galaxy, located at a distance of 7.72 Mpc (Anand et al. 2018) and containing three previously detected ULXs within its spiral arms (Liu \& Bregman 2005), including the well-studied soft and variable NGC 6946 X-1 (Holt et al. 2003; Roberts \& Colbert 2003; Fridriksson et al. 2008; Rao et al. 2010; Berghea \& Dudik 2012; Berghea et al. 2013), referred to as ULX-3 in this paper, as in Liu \& Bregman (2005), to prevent confusion with ULX-1. ULX-3 is also an ultraluminous ultraviolet source (Kaaret et al. 2010), is associated with the optical nebula MF16 (Matonick \& Fesen 1997; Abolmasov et al. 2008), and shows evidence of emission lines that may be consistent with collisional heating due to an outflowing wind (Pinto et al. 2016). It is often referred to in the literature as the only ULX of NGC 6946, as the others are less frequently observed at ULX luminosities. However, NGC 6946 also contains the soft sources NGC 6946 ULX-1 (Devi et al. 2008; Earnshaw \& Roberts 2017) and ULX-2 (Liu \& Bregman 2005) that have previously been observed as ULXs.

In this paper we report on the known ULXs in NGC 6946, including ULX-3, which we can study with broadband X-ray data for the first time. We also report on the appearance (and later disappearance) of a new source that we call ULX-4, and perform multiwavelength analysis of the region to attempt to discern its nature.

\section{Data Reduction and Analysis}

In 2017, NGC 6946 was observed twice by NuSTAR as a target of opportunity (ToO) observation in order to study the type II-P supernova SN2017eaw north of the galaxy (B. Grefenstette et al. 2019, in preparation). The two observations were taken 11 days apart, with the second observation being simultaneous with an observation by XMM-Newton. During these observations, all three previously identified ULXs were detected by XMM-Newton, and ULX-3 was detected by NUSTAR. In the second, simultaneous epoch a fourth ULX was detected by both XMM-Newton and NuSTAR, having newly appeared in the 11 days between the observations. We present a list of the ULXs in NGC 6946 in Table 1.

In our analysis of the ULXs in NGC 6946, we primarily use the two NUSTAR observations and the associated XMM-Newton observation simultaneous with the second NuSTAR observation, all taken in 2017 as part of the same ToO campaign. In examining the history of ULX-4, we also make use of all archival Chandra observations, archival XMM-Newton observations, and all Swift observations taken in 2017 . We present a list of the X-ray observations used in this study in Table 2, with the 2017 ToO observations marked in bold. We show images of the 2017 XMM-Newton and NUSTAR observations in Figure 1, along with an optical image of NGC 6946.
Table 1

Four ULXs in NGC 6946

\begin{tabular}{lccc}
\hline \hline Name $^{\mathrm{a}}$ & Position (J2000) & NuSTAR ${ }^{\mathrm{b}}$ & Other Names \\
\hline ULX-1 & $20: 35: 00.3+60: 09: 07$ & $\mathrm{~N}$ & $\ldots$ \\
ULX-2 & $20: 34: 36.5+60: 09: 30$ & $\mathrm{~N}$ & $\ldots$ \\
ULX-3 & $20: 35: 00.7+60: 11: 31$ & $\mathrm{Y}$ & $\mathrm{X}-1^{\mathrm{c}}, \mathrm{MF}^{\mathrm{d}}{ }^{\mathrm{d}}$ \\
ULX-4 & $20: 34: 56.9+60: 08: 13$ & $\mathrm{Y}$ & $\ldots$ \\
\hline
\end{tabular}

Notes.

${ }^{a}$ The source name used in this paper is as defined in Liu \& Bregman (2005) for the first three ULXs.

b Detected by $N U S T A R$

${ }^{\mathrm{c}}$ Fabbiano \& Trinchieri (1987), most commonly used name.

d Associated nebula (Matonick \& Fesen 1997).

\subsection{NuSTAR}

We reprocessed the NuSTAR observations using the NuSTAR Data Analysis Software (NuSTARDAS; v1.7.1) routine nupipeline. We extracted source and background spectra and light curves from the NUSTAR science data with the NuSTARDAS task nuproducts, using circular source extraction regions with radius $45^{\prime \prime}$ and background regions of radius $60^{\prime \prime}$ on the same chip. Spectra were grouped into 20 counts per bin to allow for $\chi^{2}$ statistics to be used in spectral model fitting. A $3 \sigma$ upper limit for the initial non-detection of ULX-4 was found using the FTOOLS task sosta, using a $45^{\prime \prime}$ radius source region and a $60^{\prime \prime}$ radius background region.

\subsection{XMM-Newton}

The XMM-Newton data were reduced using v16.1.0 of the $X M M-N e w t o n$ Science Analysis System (SAS) software and up-to-date CALDB as of 2018 June, producing calibrated event files with epproc and emproc and removing periods of background flaring-several observations were heavily affected by background flaring so they were removed from this analysis. We extracted data products using $20^{\prime \prime}$ radius circular source regions and $40^{\prime \prime}$ radius circular background regions located on the same chip at a similar distance from the readout node. We selected $\mathrm{FLAG}=0$ \&\& PATTERN $<4$ events for the EPIC-pn camera, and PATTERN $<12$ for the EPIC-MOS cameras. In all cases, spectra were grouped into 20 counts per bin as for NUSTAR. Redistribution matrices and auxiliary response files were generated with the tasks $r m f g e n$ and arfgen, respectively. $3 \sigma$ flux upper limits for nondetections were determined using the SAS task eregionanalyse, again using $20^{\prime \prime}$ radius circular source regions and $40^{\prime \prime}$ radius circular background regions.

\subsection{Chandra}

Previous Chandra observations of NGC 6946 were reduced using the task chandra_repro of v4.7.7 of the Chandra Interactive Analysis of Observations (CIAO) software. The srcflux task was used to find the $0.3-10 \mathrm{keV} 3 \sigma$ flux upper limits of non-detections, using a $3^{\prime \prime}$ radius source region surrounded by a $20^{\prime \prime}$ radius annulus for the background.

\subsection{Swift}

Swift monitored NGC 6946 closely during 2017, and we use all observations from that year. X-ray source products were generated using the FTOOLS task xrtpipeline. The 
Table 2

2017 and Archival X-Ray Observations of NGC 6946

\begin{tabular}{|c|c|c|c|}
\hline Obs. ID & Mission & Obs. Date & $\operatorname{Exposure}^{\mathrm{a}}(\mathrm{ks})$ \\
\hline \multicolumn{4}{|c|}{2017 ToO observations } \\
\hline 90302004002 & NuSTAR & 2017 May 21 & 66.7 \\
\hline 90302004004 & NuSTAR & 2017 Jun 1 & 47.7 \\
\hline 0794581201 & XMM-Newton & 2017 Jun 1 & 44.0/45.2/39.2 \\
\hline 0200670101 & XMM-Newton & 2004 Jun 9 & $4.0 / 4.1 / 0.8$ \\
\hline 0200670301 & XMM-Newton & 2004 Jun 13 & $12.4 / 12.4 / 8.3$ \\
\hline 0200670401 & XMM-Newton & 2004 Jun 25 & $8.6 / 9.4 / 0.8$ \\
\hline 0401360201 & XMM-Newton & 2006 Jun 2 & $4.6 / 4.4 / 0.1$ \\
\hline 0401360301 & XMM-Newton & 2006 Jun 18 & $6.0 / 6.0 / 1.7$ \\
\hline 0500730101 & XMM-Newton & 2007 Nov 8 & $27.9 / 28.3 / 20.2$ \\
\hline 0500730201 & XMM-Newton & 2007 Nov 2 & $32.4 / 32.6 / 29.8$ \\
\hline 0691570101 & XMM-Newton & 2012 Oct 21 & $110.7 / 112.2 / 98.2$ \\
\hline 1043 & Chandra & 2001 Sep 7 & 58.3 \\
\hline 4404 & Chandra & 2002 Nov 25 & 30.0 \\
\hline 4631 & Chandra & 2004 Oct 22 & 29.7 \\
\hline 4632 & Chandra & 2004 Nov 6 & 28.0 \\
\hline 4633 & Chandra & 2004 Dec 3 & 26.6 \\
\hline 13435 & Chandra & 2012 May 21 & 20.4 \\
\hline 17878 & Chandra & 2016 Sep 28 & 40.0 \\
\hline 19887 & Chandra & 2016 Sep 28 & 18.6 \\
\hline 19040 & Chandra & 2017 Jun 11 & 9.8 \\
\hline 00010130001 & Swift-XRT & 2017 May 14 & 2.0 \\
\hline 00010130003 & Swift-XRT & 2017 May 15 & 1.8 \\
\hline 00010130004 & Swift-XRT & 2017 May 15 & 3.8 \\
\hline 00010130005 & Swift-XRT & 2017 May 16 & 1.7 \\
\hline 00010130006 & Swift-XRT & 2017 May 17 & 2.6 \\
\hline 00010130007 & Swift-XRT & 2017 May 18 & 1.5 \\
\hline 00010130008 & Swift-XRT & 2017 May 22 & 4.0 \\
\hline 00010130010 & Swift-XRT & 2017 May 24 & 1.7 \\
\hline 00010130011 & Swift-XRT & 2017 May 26 & 1.8 \\
\hline 00010130012 & Swift-XRT & 2017 May 29 & 0.7 \\
\hline 00010130013 & Swift-XRT & 2017 May 29 & 1.3 \\
\hline 00010130014 & Swift-XRT & 2017 May 31 & 1.5 \\
\hline 00010130015 & Swift-XRT & 2017 Jun 2 & 1.5 \\
\hline 00010130016 & Swift-XRT & 2017 Jun 4 & 1.5 \\
\hline 00010130017 & Swift-XRT & 2017 Jun 6 & 1.6 \\
\hline 00010130018 & Swift-XRT & 2017 Jun 8 & 1.4 \\
\hline 00010130019 & Swift-XRT & 2017 Jun 10 & 1.5 \\
\hline 00010130020 & Swift-XRT & 2017 Jun 12 & 0.5 \\
\hline 00010130021 & Swift-XRT & 2017 Jun 14 & 1.1 \\
\hline 00010130022 & Swift-XRT & 2017 Jun 16 & 1.5 \\
\hline 00010130023 & Swift-XRT & 2017 Jul 15 & 3.0 \\
\hline 00010130024 & Swift-XRT & 2017 Jul 28 & 3.0 \\
\hline 00010130025 & Swift-XRT & 2017 Aug 11 & 1.0 \\
\hline 00010130026 & Swift-XRT & 2017 Aug 16 & 1.3 \\
\hline 00010130027 & Swift-XRT & 2017 Aug 25 & 3.2 \\
\hline 00010130028 & Swift-XRT & 2017 Sep 8 & 1.9 \\
\hline 00010130029 & Swift-XRT & 2017 Sep 13 & 0.9 \\
\hline
\end{tabular}

Note.

${ }^{\text {a }}$ Given as EPIC MOS1/MOS2/pn for XMM-Newton, after removal of periods of background flaring.

spectrum for ULX-3 was taken using a $45^{\prime \prime}$ radius source region and a $70^{\prime \prime}$ radius background region located outside of the galaxy, and the spectrum was grouped to 20 counts per bin as for NuSTAR. $3 \sigma$ flux upper limits for non-detections in Swift observations were determined using the FTOOLS task sosta, using a $20^{\prime \prime}$ radius source region (to avoid contamination by a nearby source) and a $70^{\prime \prime}$ radius background region as for ULX-
3. Magnitude lower limits for optical/ultraviolet non-detections were determined using the FTOOLS task uvotsource.

\subsection{Hubble Space Telescope (HST)}

Optical photometry was performed on five observations of NGC 6946 taken using the WFPC2 and WFC3/IR cameras on board the HST during three different epochs before 2017, which cover the region of the galaxy in which ULX-4 is contained. We use observations in the F547M, F606W, and F814W optical bands with WFPC2 (proposal IDs 8591, 8597, and 8599 , respectively; the F656N band is also observed, but the resolution is insufficiently good to characterize individual sources in this band) and the F110W and F128N near-infrared bands with WFC3/IR (proposal ID 14156). We retrieved preprocessed images from the Hubble Legacy Archive created from multiple exposures combined using the MultiDrizzle routine.

We corrected the HST astrometry using the USNO star catalog and the Image Reduction and Analysis Facility tools ccfind, ccmap and ccupdatewcs, and combined the $90 \%$ confidence error circle of the resulting HST position errors in quadrature with the XMM-Newton source position error, resulting in a $0.1990 \%$ error circle around the source position, which we used to identify potential counterparts. We performed aperture and point-spread function (PSF)-fitting photometry on these potential optical counterparts using the DAOPHOT II/ ALLSTAR software package (Stetson 1987), using the values from aperture photometry where a good PSF fit could not be found, and placed magnitude limits based on a combination of the read noise, dark current, and sky background where a source could not be detected at all.

\section{Results and Discussion}

All three previously identified ULXs in NGC 6946 are detected by XMM-Newton, with ULX-3 also detected by NuSTAR. ULX-1 and ULX-2 were both at sub-ULX luminosities during the simultaneous XMM-Newton and NuSTAR observation, and only detected with $X M M-N e w t o n$, although we briefly characterize them for completeness. Both XMMNewton and NUSTAR also detect a new ULX, which we call ULX-4, at 20:34:56.7+60:08:12. In this section we present our analysis and a discussion of these four objects. We perform all spectral fitting using v12.10 of the XSPEC (Arnaud 1996) software, and all quoted models are given in XSPEC syntax. Uncertainties are given at the $90 \%$ confidence level, and we use the abundance tables of Wilms et al. (2000) throughout.

\subsection{NGC $6946 U L X-1$ and ULX-2}

We fitted the XMM-Newton spectra of ULX-1 and ULX-2 from observation 0794581201 with an absorbed power-law model, using two tbabs absorption components, one frozen to the Galactic value of $N_{\mathrm{H}}=1.84 \times 10^{21} \mathrm{~cm}^{-2}$ and the other allowed to vary. In the case of ULX-1, a power-law model was not sufficient to produce a good fit, with $\chi^{2} /$ dof $=131.9 / 90$ and the fit showing significant soft residuals at $\sim 1 \mathrm{keV}$. These soft residuals are known to be a common feature in the spectra of ULXs and bright X-ray binaries (e.g., Bauer \& Brandt 2004; Carpano et al. 2007; Middleton et al. 2015b), and found in other ULXs to be a combination of emission and absorption features related to powerful outflowing winds (including in NGC 6946 ULX-3; Pinto et al. 2016). We used an additional 

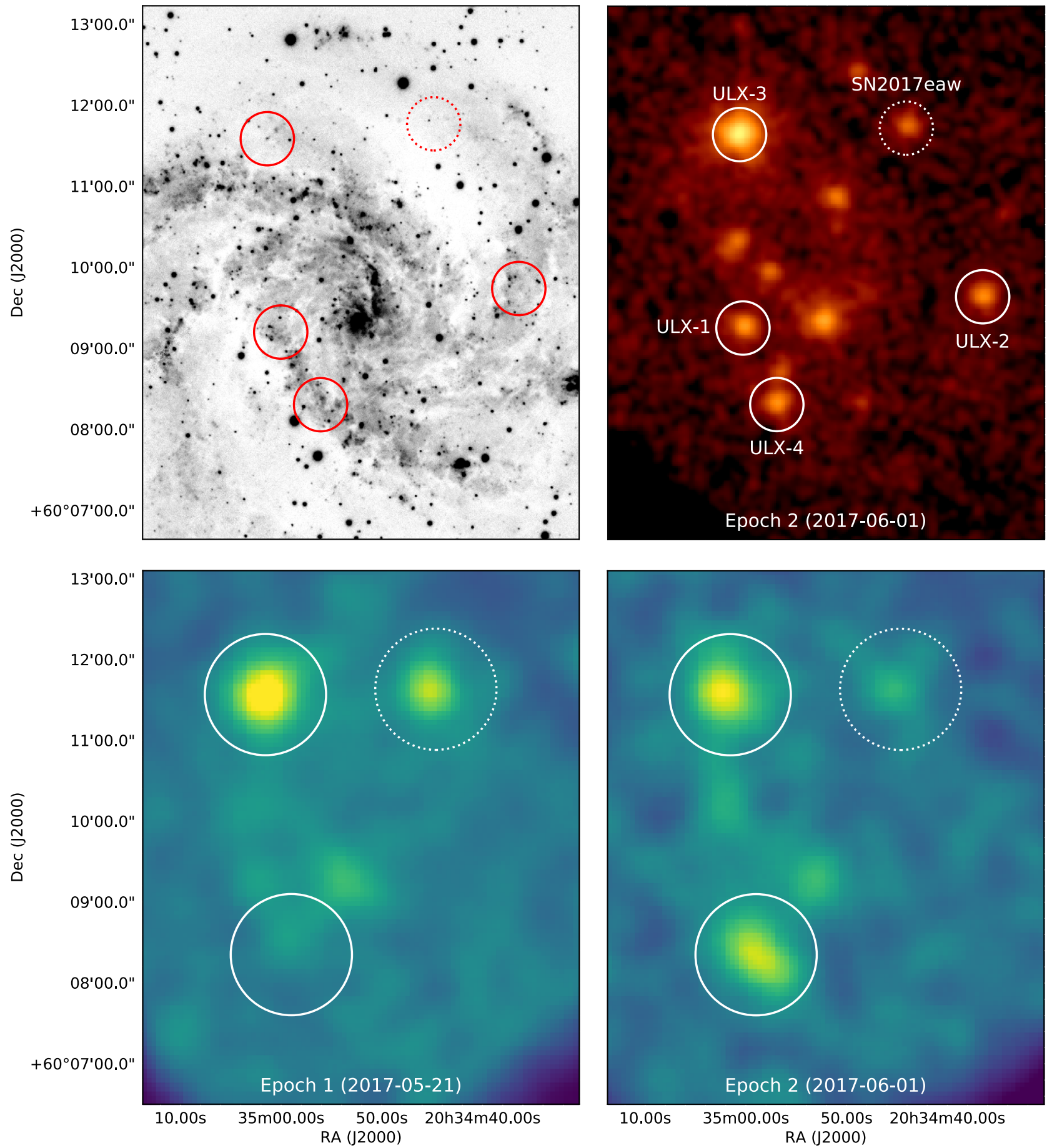

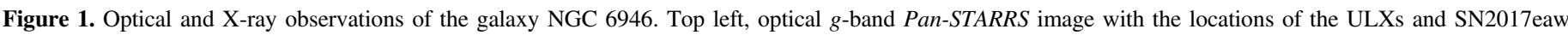

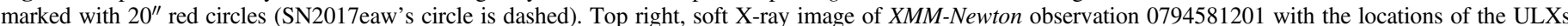

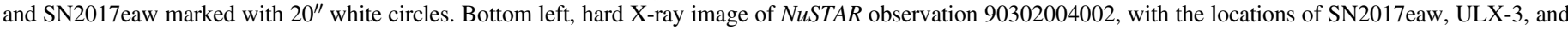

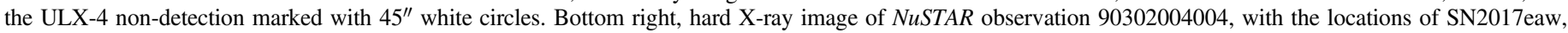
ULX-3, and ULX-4 marked with $45^{\prime \prime}$ white circles.

Gaussian component to empirically fit these soft residuals, which resulted in a very large statistical improvement and provided an acceptable fit $\left(\chi^{2} /\right.$ dof $\left.=84.5 / 87\right)$. Using the bestfitting model for each source, we calculated the absorbed 0.3-10 keV flux of both objects. We do not correct for absorption since extending steep power laws, which are empirical rather than physical models, to low energies is likely to overestimate the true flux of the object beneath the absorption-unabsorbed fluxes using such models can be a factor of 2 or 3 higher than those for more physically motivated models, and the amount of absorption is itself modeldependent, so we make fewer assumptions by just considering the observed, absorbed flux. For comparison, we also fitted both spectra with an absorbed multicolor disk model. Although this was able to produce a statistically acceptable fit, the Gaussian representing the soft residuals in ULX-1 contributes 
Table 3

The ULX-1 and ULX-2 Spectral-fitting Results for XMM-Newton Observation 0794581201

\begin{tabular}{|c|c|c|c|c|c|c|c|c|}
\hline Source & $\begin{array}{c}N_{\mathrm{H}}^{\mathrm{a}} \\
\left(10^{21} \mathrm{~cm}^{-2}\right)\end{array}$ & $\begin{array}{l}\Gamma / T_{\text {in }} \\
(-/ \mathrm{keV})\end{array}$ & $p^{\mathrm{b}}$ & $\begin{array}{c}T_{\text {in }} \\
(\mathrm{keV})\end{array}$ & $\begin{array}{c}E_{\text {line }} \\
(\mathrm{keV})\end{array}$ & $\begin{array}{c}\sigma_{\text {line }} \\
(\mathrm{keV})\end{array}$ & $\chi^{2} /$ dof & $\begin{array}{c}F_{0.3-10 \mathrm{kev}^{\mathrm{c}}} \\
\left(10^{-14} \mathrm{erg} \mathrm{s}^{-1}\right)\end{array}$ \\
\hline \multirow[t]{6}{*}{ ULX-1 } & tbabs*tbabs $*$ & (powerlaw & \multicolumn{6}{|c|}{ + Gauss) } \\
\hline & $4.2_{-0.6}^{+0.7}$ & $4.5 \pm 0.3$ & $\cdots$ & $\cdots$ & $\cdots$ & $\cdots$ & $131.9 / 90$ & $\cdots$ \\
\hline & $2.3_{-0.7}^{+0.8}$ & $3.6_{-0.3}^{+0.4}$ & $\cdots$ & $\cdots$ & $0.90_{-0.07}^{+0.04}$ & $0.13_{-0.04}^{+0.05}$ & $84.5 / 87$ & $9.8 \pm 0.4$ \\
\hline & tbabs*tbabs* & (diskbb & \multicolumn{6}{|c|}{ + Gauss) } \\
\hline & $0.6 \pm 0.4$ & $0.31 \pm 0.03$ & $\ldots$ & $\cdots$ & $\ldots$ & $\ldots$ & $179.7 / 90$ & $\cdots$ \\
\hline & $<8.2$ & $0.6 \pm 0.1$ & $\cdots$ & $\cdots$ & $0.7 \pm 0.1$ & $0.29_{-0.05}^{+0.06}$ & $99.1 / 87$ & ... \\
\hline \multirow[t]{5}{*}{ ULX-2 } & $\begin{array}{c}\text { tbabs } * \text { tbabs } * \\
2.9_{-1.0}^{+1.1} \\
\text { tbabs } * \text { tbabs } *\end{array}$ & $\begin{array}{c}\text { powerlaw } \\
3.6_{-0.3}^{+0.4} \\
\text { (diskbb }\end{array}$ & $\cdots$ & $\begin{array}{c}\cdots \\
+ \text { diskbb) }\end{array}$ & $\cdots$ & $\cdots$ & $44.9 / 42$ & $7.8 \pm 0.5$ \\
\hline & $<0.3$ & $0.43 \pm 0.04$ & $\cdots$ & $\cdots$ & $\cdots$ & $\cdots$ & $62.6 / 42$ & $\cdots$ \\
\hline & $3.3_{-0.2}^{+0.3}$ & $0.17_{-0.04}^{+0.06}$ & $\cdots$ & $0.7_{-0.1}^{+0.2}$ & $\cdots$ & $\cdots$ & $38.1 / 40$ & $\cdots$ \\
\hline & tbabs*tbabs* & dis & & & & & & \\
\hline & $<0.8$ & $0.7 \pm 0.1$ & $<0.56$ & $\cdots$ & $\cdots$ & ... & $52.6 / 41$ & $\ldots$ \\
\hline
\end{tabular}

Notes.

${ }^{\mathrm{a}}$ The column density for the absorption component allowed to vary, with the first component frozen to the Galactic value of $N_{\mathrm{H}}=1.84 \times 10^{21} \mathrm{~cm}^{-2}$.

$\mathrm{b}$ The radial dependence of disk temperature, with a hard lower limit of 0.5 .

c The absorbed flux in the $0.3-10 \mathrm{keV}$ band.

to a physically unreasonable portion of the spectrum, broadening to fit the majority of the soft emission, and the residuals for ULX-2 are even less well characterized than for a powerlaw fit. Therefore, a steep power law seems to be the best empirical model for both spectra. We present the spectral fit results for these two sources in Table 3 and the best-fitting model plots in Figure 2.

Both sources are found to be very soft and at a low enough flux to be a little below the ULX threshold luminosity (both have $L_{\mathrm{X}} \sim 6-7 \times 10^{38} \mathrm{erg} \mathrm{s}^{-1}$ ), consistent with these sources being persistently bright Eddington threshold objects that only occasionally reach the ULX luminosity regime. ULX-1 is consistent with a steep power-law spectral shape with soft residuals, as previously found in Earnshaw \& Roberts (2017), where it was suggested that it is a super-Eddington accreting source viewed at a high accretion rate and/or at a high inclination, with most of the high-energy central emission obscured and down-scattered by the surrounding wind and outer disk. While the most typical examples of ultraluminous supersoft sources have spectra that are almost entirely dominated by thermal emission (e.g., Urquhart \& Soria 2016), the very steep power-law tail may come from a very small amount of visible central emission, making ULX-1 a source on the borderline between the soft ultraluminous regime and the thermal-dominated ultraluminous supersoft sources. ULX-2 lies on an EPIC-pn chip gap, so we only have data from the EPIC-MOS cameras. Its spectrum, while similarly steep to ULX-1 and statistically well fitted by a simple absorbed power law, appears on visual inspection to have a two-component shape to its residuals not dissimilar to that found in the soft ultraluminous accretion regime in ULXs (e.g., Gladstone et al. 2009). However, likely owing to the low signal-to-noise and limited bandpass, fitting the spectrum with two thermal components rather than a power law does not provide a significant improvement in the quality of fit. Still, it is possible that this source is also a super-Eddington accreting system like ULX-1, only with typical luminosity below the standard ULX definition.

\subsection{NGC $6946 U L X-3$}

We began by fitting the combined XMM-Newton and NUSTAR spectrum of ULX-3, with the cross calibration fixed to unity, with an absorbed power-law model as for ULX-1 and ULX-2. Like ULX-1, its residuals are dominated by the likely wind-related feature at $\sim 1 \mathrm{keV}$, so we fitted this feature with a Gaussian component as before, which yields a statistically acceptable fit to the spectrum $\left(\chi^{2} /\right.$ dof $\left.=332.8 / 289\right)$. With a photon index of $\Gamma=2.51 \pm 0.05$, ULX-3 is the softest ULX with a broadband X-ray spectrum detected by NuSTAR studied so far. We test for a spectral turnover at high energies as seen in other ULXs, a key indicator of super-Eddington accretion. However, we find that there is no significant improvement in fit with a cutoff power-law model over a power law without a cutoff ( $\Delta \chi^{2}=1.2$ for 1 dof). We simulated 1000 spectra with the cutoff power-law parameters we measure, and found that for a spectrum this soft, NUSTAR is unable to significantly detect a cutoff and correctly constrain its parameters. We show the best-fitting model parameters for the different models in Table 4 and show the spectrum and residuals in Figure 3.

We used the best-fitting power-law model to calculate the absorbed $0.3-10 \mathrm{keV}$ flux of ULX-3 during this observation, which we find to be $(9.1 \pm 0.2) \times 10^{-13} \mathrm{erg} \mathrm{cm}^{-2} \mathrm{~s}^{-1}$, corresponding to a luminosity of $(6.5 \pm 0.1) \times 10^{39} \mathrm{erg} \mathrm{s}^{-1}$. This is broadly consistent with previously calculated luminosities for this source when accounting for lower distances to the galaxy used previously (e.g., Middleton et al. 2015a), and it therefore continues to be a persistent ULX.

While there is no statistical case for a more complex model, we do consider the scenario that this ULX has a similar spectrum to other ULXs observed with NUSTAR in the past and fit it with a typical diskbb+diskpbb model-the soft diskbb component originating in an outer thin disk/soft outflowing wind, and the harder diskpbb component originating in the geometrically thick inner disk (Walton et al. 2014; Middleton et al. 2015a). We find that fitting the spectrum in this manner yields an acceptable fit $\left(\chi^{2} /\right.$ dof $\left.=324.8 / 286\right)$, but also hints at a hard excess as often seen in hard ULXs at energies $>20 \mathrm{keV}$ (Walton et al. 

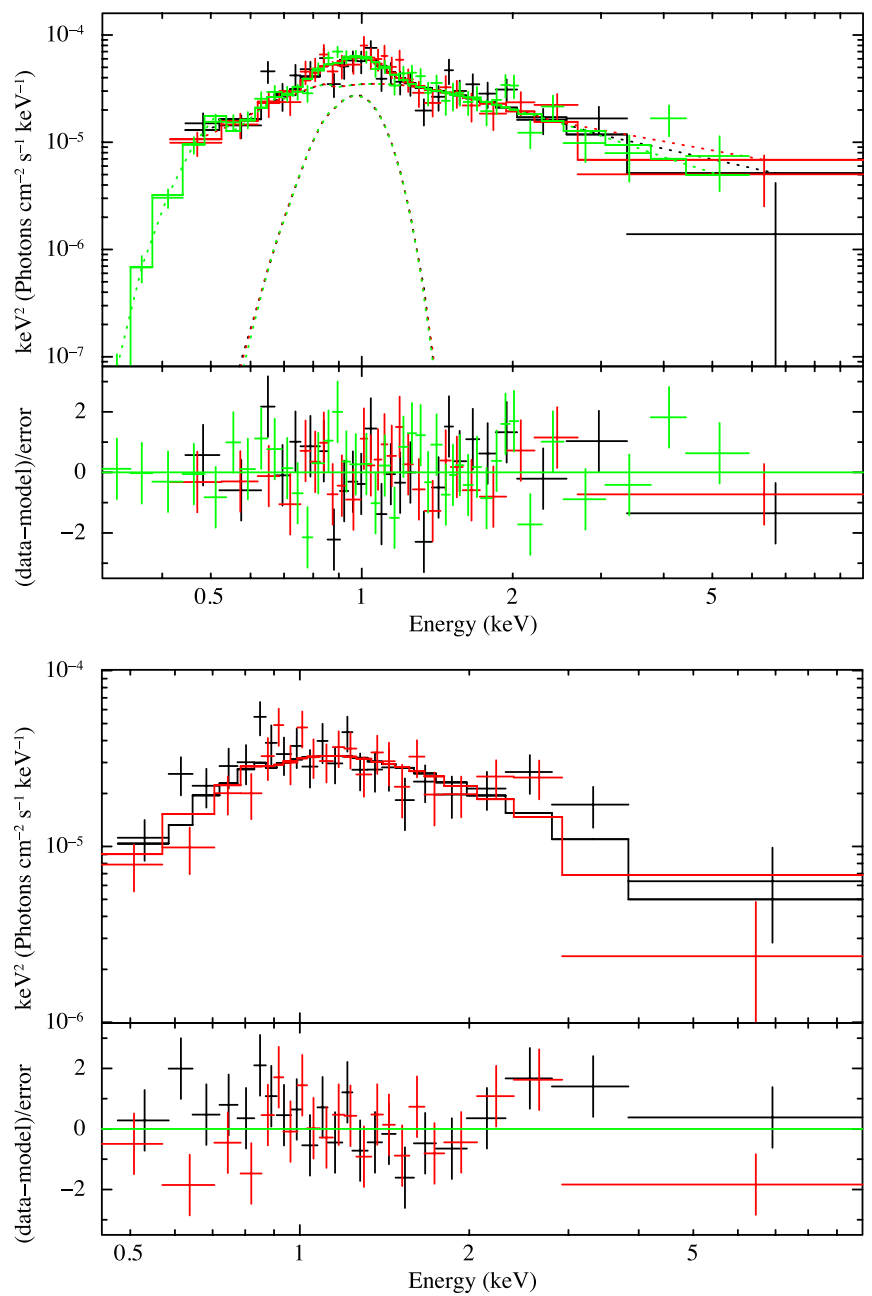

Figure 2. The XMM-Newton observation 0794581201 spectrum and residuals for ULX-1 (top) and ULX-2 (bottom). Black, red, and green data points are EPIC MOS1, MOS2, and pn data, respectively-ULX-2 lies on a pn chip gap, so only MOS data is available. ULX-1 is plotted with the best-fitting absorbed power-law and Gaussian model with $\Gamma=3.6_{-0.3}^{+0.4}$ and $E_{\text {line }}=0.90_{-0.07}^{+0.04} \mathrm{keV}$. ULX-2 is plotted with the best-fitting absorbed power-law model with $\Gamma=3.6_{-0.3}^{+0.4}$. Full fit details are provided in Table 3 .

2014, 2015b; Mukherjee et al. 2015), which cannot be entirely accounted for by a maximally broadened-disk component at high energies. This hard excess feature is not significant in these observations of ULX-3, but if it is the case that this source is spectrally similar to other ULXs with an excess present, this may be an additional reason why we do not see evidence of a turnover in the spectrum, as the entire spectrum is sufficiently soft that the strength of a turnover feature would be counteracted by a steep power-law excess at the energies we observe.

There is no simultaneous XMM-Newton observation for the first $N U S T A R$ observation, though there is a Swift observation the following day, so we fit these observations together to get our best possible picture of the broadband spectrum during the first NuSTAR epoch. The amount of Swift data is insufficient to determine whether the soft residuals that we fitted with a Gaussian in the XMM-Newton data are contributing to the soft emission in the first observation as well, so the best comparison we can make is to the power-law-only fit to the second observation. We find that both the flux and spectral hardness are consistent with the second epoch, with no evidence for any significant variability on a ten-day timescale. While the NUSTAR data appears to hint at a spectral turnover at $\sim 8 \mathrm{keV}$ on a visual inspection of the residuals, a cutoff power law offers only a small improvement in $\chi^{2}$ over a regular powerlaw model $\left(\Delta \chi^{2}=3.4\right.$ for 1 dof $)$. We show the best-fitting model parameters in Table 4 and show the spectrum and residuals in Figure 4.

Our weaker constraints on the soft part of the spectrum in the first epoch limit our ability to comment on any variability of ULX-3 between the two observations. However, we can investigate the short-term variability of ULX-3 by creating an EPIC-pn power spectrum for the XMM-Newton observation, averaging the periodograms of 70 segments of length $601 \mathrm{~s}$ ( i.e., 8192 time bins at the $73.4 \mathrm{~ms}$ time resolution of the EPICpn instrument). We find the power spectrum to exhibit red noise, as expected to be found in accreting systems (see Figure 5), but find no evidence for any quasi-periodic oscillations (QPOs) at $\sim 10^{-2} \mathrm{~Hz}$ like those reported for earlier observations in Rao et al. (2010). We can rule out the presence of a $8.5 \mathrm{mHz}$ QPO, as previously observed, at the $5 \sigma$ level for this observation. We also do not significantly detect any other QPOs in the power spectrum. However, the fractional rms variability of ULX-3 is $40 \% \pm 4 \%$ over the $1-100 \mathrm{mHz}$ range, showing that ULX-3 continues to be highly variable and its spectrum remains bright and steep as it has previously been observed, indicating that the QPOs are possibly a transient feature in an otherwise persistent accretion state.

\subsection{NGC $6946 U L X-4$}

ULX-4 is a new transient source. It was not detected in the first NuSTAR observation, but was strongly detected by both telescopes in the second joint XMM-Newton and NuSTAR observation.

As for the other sources, we fitted the combined $X M M$ Newton and NuSTAR spectrum of ULX-4 with an absorbed power-law model. We found that no absorption component is required in addition to the Galactic column, so our models for this source only contain a single tbabs component frozen to the Galactic value, which is somewhat unusual for ULXs, which usually show evidence of a local absorption column. This source has a very hard $(\Gamma \sim 1)$ power-law-shaped spectrum, and the fit is greatly improved $\left(\Delta \chi^{2}=15.8 / 1\right.$ dof $)$ by the presence of a cutoff at $\sim 11 \mathrm{keV}$. We checked the significance of this cutoff by simulating a power-law spectrum 10,000 times and finding the improvement in $\chi^{2}$ of a cutoff power-law model compared to a power law due to random fluctuations in the spectrum. We found that $0.02 \%$ of simulations had $\Delta \chi^{2} \geqslant 15.8$, making the cutoff that we detect in the source spectrum significant to $\sim 3.5 \sigma$. The spectrum can also be well fitted with a very hot multicolor disk blackbody model with $k T_{\text {in }}=4.3_{-0.5}^{+0.6} \mathrm{keV}$, with no need for broadening using a diskpbb model - although over the portion of the spectrum we see, this is functionally very similar to the cutoff power-law model. We find the time-averaged $0.3-10 \mathrm{keV}$ flux to be $3.2 \times 10^{-13} \mathrm{erg} \mathrm{cm}^{-2} \mathrm{~s}^{-1}$, placing this new source just inside the ULX regime at $L_{\mathrm{X}}=(2.27 \pm 0.07) \times 10^{39} \mathrm{erg} \mathrm{s}^{-1}$. We show the joint XMM-Newton and NUSTAR spectrum in Figure 6, and the spectral-fitting results for ULX-4 in Table 5.

We examined Swift-XRT data both immediately before, between, and after the two NuSTAR observations, as well as into the remainder of 2017. Due to the short observations and the hardness of the source spectrum, ULX-4 is not detected in 
Table 4

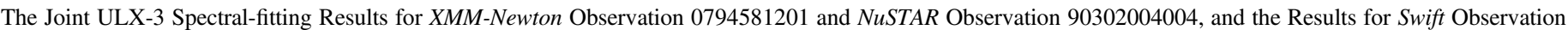
00010130008 and NUSTAR Observation 90302004002

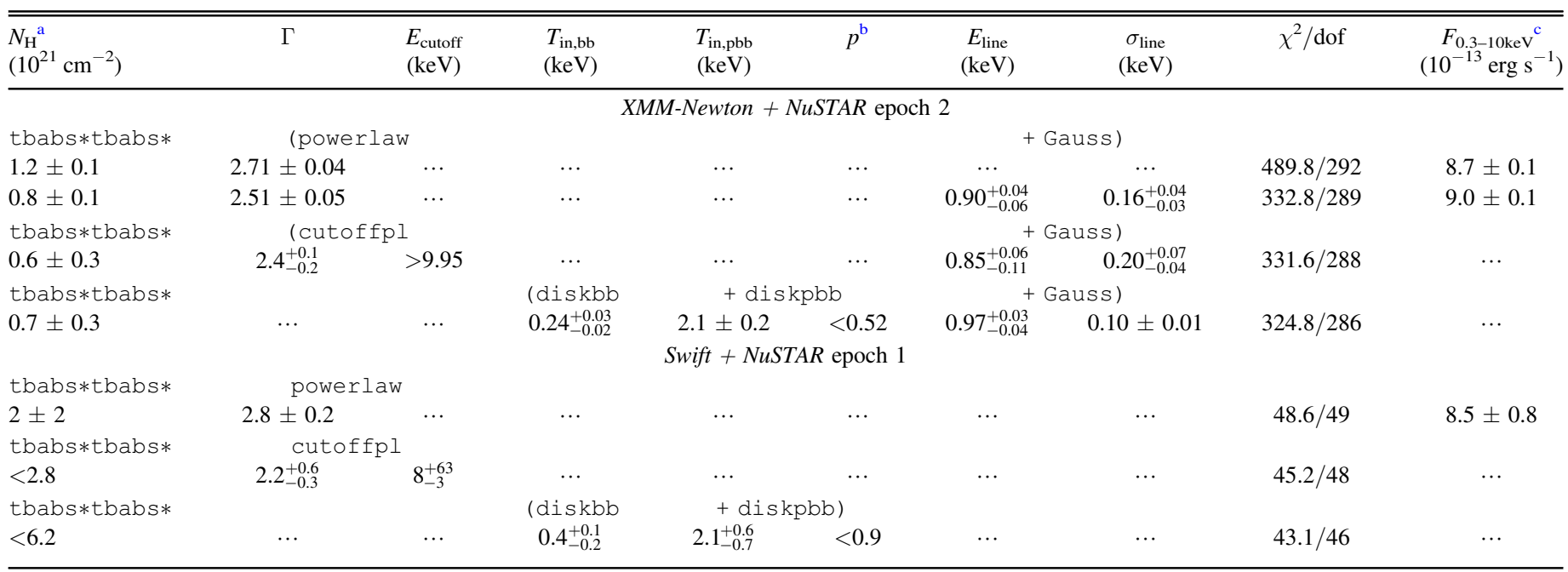

Notes.

a The column density for the absorption component allowed to vary, as in Table 3.

b The radial dependence of disk temperature, as in Table 3.

c The absorbed flux in the $0.3-10 \mathrm{keV}$ band.

any of these observations and we are unable to place strong limits on the $0.3-10 \mathrm{keV}$ flux during the period over which the source appears beyond that it undergoes at least a factor $>1.4$ increase in flux between the two NuSTAR epochs. Similarly, putting an upper limit on the $3-20 \mathrm{keV}$ flux from the initial NUSTAR

non-detection $\left(F_{3-20 \mathrm{keV}}<6.23 \times 10^{-14} \mathrm{erg} \mathrm{cm}^{-2} \mathrm{~s}^{-1}\right)$ gives us a factor $>5$ increase in flux for that energy band.

In order to confirm that the detection by XMM-Newton and $N U S T A R$ is the first appearance of ULX-4, we plot a long-term light curve from archival XMM-Newton and Chandra observations (Figure 7), assuming a similar spectral shape to the one we observe here of $\Gamma=1$ and Galactic absorption. This assumption gives conservative flux upper limits; should the source have previously been in a softer state, these upper limits would be lower. We find no previous detections of ULX-4Chandra observations place the strongest limits on the $0.3-10 \mathrm{keV}$ flux, with each upper limit below $F_{\mathrm{X}}<3.5 \times 10^{-15} \mathrm{erg} \mathrm{cm}^{-2} \mathrm{~s}^{-1}$, or $L_{\mathrm{X}}<2.5 \times 10^{37} \mathrm{erg} \mathrm{s}^{-1}$. This implies a factor $\gtrsim 80$ increase in flux between its detection in 2017 and when the source was previously observed by Chandra in 2016. Ten days after the second NuSTAR epoch, a further observation with Chandra does not detect the source either, implying an upper limit on the $0.3-10 \mathrm{keV}$ flux of $F_{\mathrm{X}}<1.1 \times 10^{-14} \mathrm{erg} \mathrm{cm}^{-2} \mathrm{~s}^{-1}$, or $L_{\mathrm{X}}<7.8 \times 10^{37} \mathrm{erg} \mathrm{s}^{-1}$, a factor of $\sim 26$ below the flux of the XMM-Newton detection. Therefore this source is transient, and the outburst that we observe lasts a maximum of 20 days.

ULX-4 is highly variable in the XMM-Newton energy band over the course of the observation. Its light curve, which we examine in three energy bands (Figure 8), shows a low initial flux, a predominantly soft flare lasting $\sim 5 \mathrm{ks}$ and releasing $\sim 10^{43} \mathrm{erg}$ of energy, followed by a second increase of flux in the final $10 \mathrm{ks}$ of the observation with a corresponding softening of the spectrum (there is a gap at $\sim 3.4 \times 10^{4}$ s due to removing a period of soft proton flaring rather than source behavior). The final count rate is over an order of magnitude higher than the count rate at the start of the observation in the soft bands. The NUSTAR light curve (Figure 9) shows the source to persist throughout the remaining $50 \mathrm{ks}$ of the observation, though it continues to vary in both soft and hard energy bands.

Dividing the $50 \mathrm{ks} X M M-N e w t o n$ observation into five $10 \mathrm{ks}$ segments, we can track the spectral and flux evolution of ULX4 over the course of the observation (see Figure 10). The source begins in a relatively soft low-flux state $(\Gamma=2 \pm 1$, $F_{\mathrm{X}}=2.6 \times 10^{-14} \mathrm{erg} \mathrm{cm}^{-2} \mathrm{~s}^{-1}$ in the first $10 \mathrm{ks}$ of the observation) and quickly reaches its very hard $(\Gamma=0.9 \pm 0.3)$ power-law slope in the second interval. During the third interval, which encompasses most of the soft flare, the spectrum nonetheless remains hard $\left(\Gamma=0.8_{-0.1}^{+0.2}\right)$ and only shows signs of softening in the final interval $(\Gamma=1.2 \pm 0.2)$. At the end of the observation, the ULX-4 has a flux of $F_{\mathrm{X}}=5.0 \times 10^{-13} \mathrm{erg} \mathrm{cm}^{-2} \mathrm{~s}^{-1}$. Therefore this source undergoes a factor $\sim 20$ increase in flux and transitions into the ULX luminosity regime over the course of the observation.

We created a power spectrum for the XMM-Newton observation of ULX-4 by averaging the periodograms of 72 segments of length $601 \mathrm{~s}$. There are no significant features in the power spectrum beyond some possible red noise becoming apparent at low frequencies $\left(\lesssim 10^{-3} \mathrm{~Hz}\right)$, which is consistent with an accreting source. Similarly, we created a power spectrum for the NuSTAR observation, though no features are seen. We searched for pulsations using the High-ENergy Data Reduction Interface from the Command Shell (HENDRICS) software (Bachetti 2015), a package based on stingray (Huppenkothen et al. 2019) for timing analysis of X-ray data and particularly optimized to handle NUSTAR data. We ran a pulsation search, considering potential acceleration with $\dot{f}$ up to $10^{-8} \mathrm{~Hz} \mathrm{~s}^{-1}$, but failed to detect any pulsations between $10^{-4}$ and $10 \mathrm{~Hz}$. On simulating light curves of ULX-4, assuming a sinusoidal pulsation with a constant $1 \mathrm{~s}$ period at different pulse fractions, we find that we can place a $90 \%$ upper 

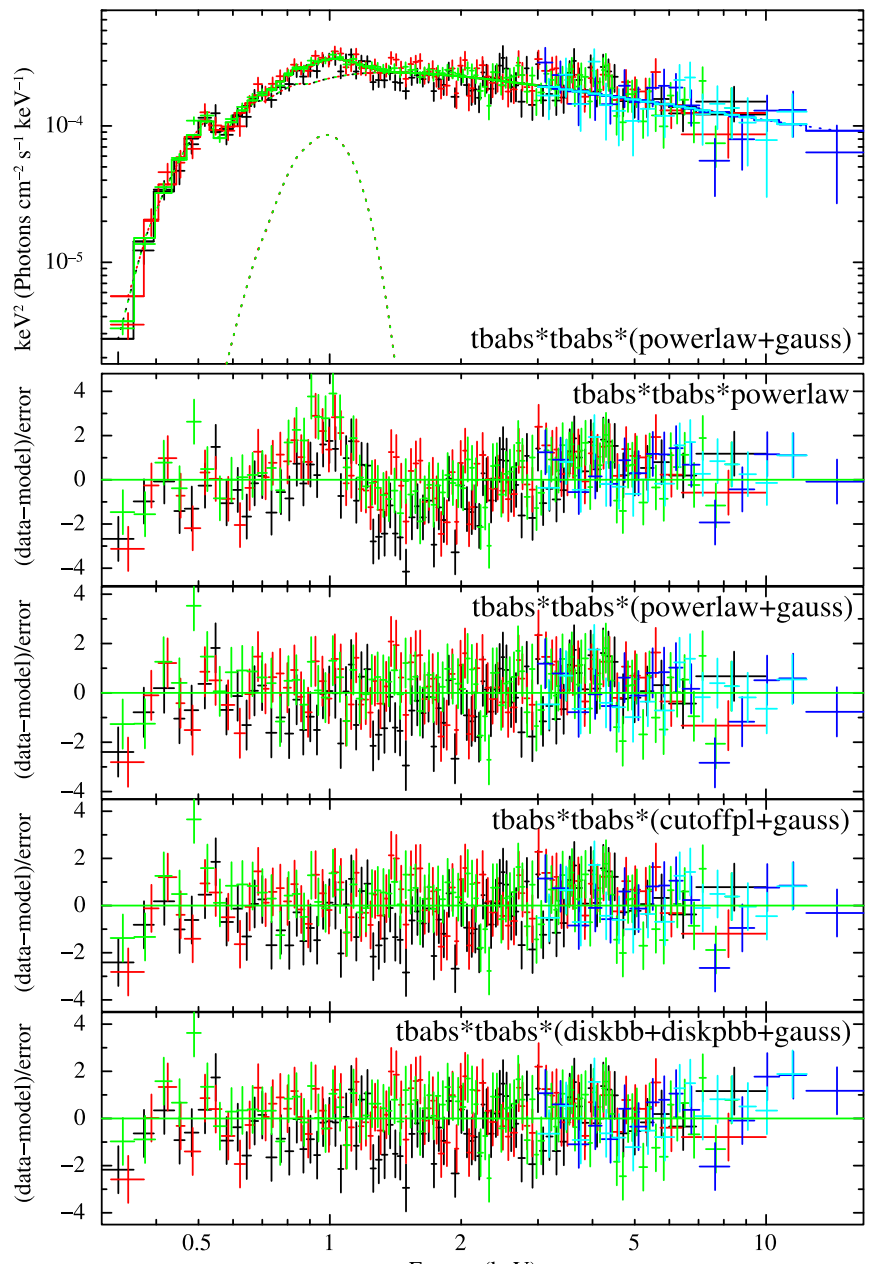
Figure 3. The XMM-Newton observation 0794581201 and NuSTAR observa-
tion 90302004004 joint spectrum of ULX-3, with XMM-Newton colors as in Figure 2 and the NuSTAR FPMA and FPMB data plotted in blue and cyan, respectively. This spectrum is plotted with the best-fitting absorbed power-law and Gaussian model, with $\Gamma=2.51 \pm 0.05$ and $E_{\text {line }}=0.90_{-0.06}^{+0.04} \mathrm{keV}$, along with the residuals for four models used to fit the spectrum as labeled in the figure.

limit on the pulsed fraction of $\sim 20 \%$ for the observed light curve. Given that ULX pulsars are known to be affected by spin-up and orbital modulations (e.g., Bachetti et al. 2014; Carpano et al. 2018), it is conceivable that we would not detect a pulsation at higher pulse fractions than this with the data we currently possess. We therefore cannot rule out the potential presence of pulsations in this source during its outburst.

There is no obvious single optical counterpart to the X-ray source. Data taken with the Ultraviolet and Optical Imager instrument on the Swift satellite show no detection during any of the Swift observations of NGC 6946, including the days immediately before and after the second NUSTAR epoch. While we have no optical data precisely simultaneous with the X-ray detection, this limits the presence of an optical counterpart with magnitude $m_{\mathrm{v}}<18.1$ and $m_{\mathrm{b}}<19.0 \quad\left(M_{\mathrm{v}}<-11.1\right.$, $\left.M_{\mathrm{b}}<-10.2\right)$ to less than a day, if one appears at all. However optical counterparts of ULXs are mostly far fainter than this (e.g., Gladstone et al. 2013), so these limits do not place strong constraints on the sort of counterpart that may be present, though they do rule out a very bright optical transient.

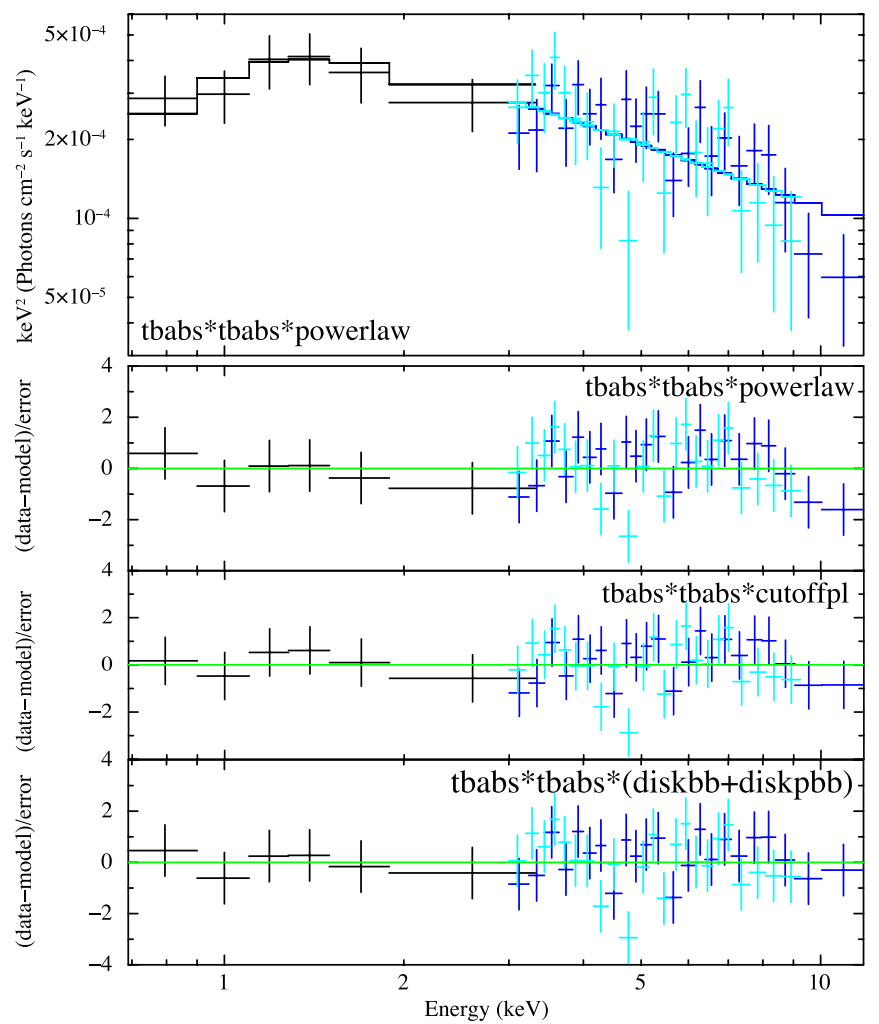

Figure 4. The Swift observation 00010130008 and NuSTAR observation 90302004002 joint spectrum of ULX-3, with Swift-XRT data in black and the NuSTAR data as in Figure 3. The spectrum is plotted with the best-fitting absorbed power-law model, with $\Gamma=2.8 \pm 0.2$, along with the residuals for three models used to fit the spectrum as labeled in the figure.

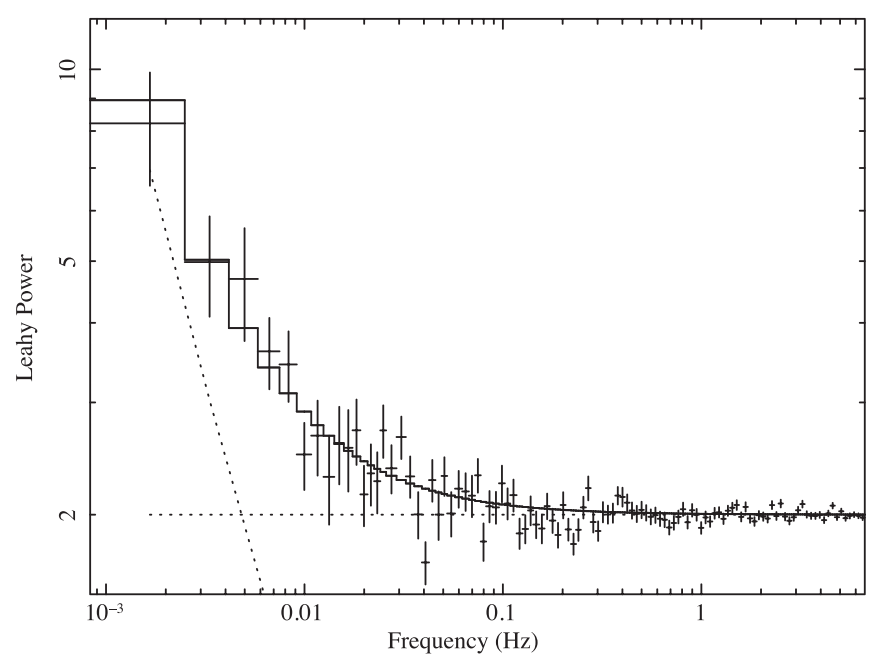

Figure 5. The EPIC-pn power spectrum for ULX-3 during observation 0794581201, plotted using Leahy normalization and fitted with a constant for the white noise level and a power-law model for the red noise.

The region of NGC 6946 in which ULX-4 is located has been observed with the $H S T$ on several occasions with the WFPC2 and WFC3/IR instruments-in the F547M, F606W, and F814W optical bands with WFPC2 and the F110W and F128N near-infrared bands with WFC3/IR. The F656N band is also observed with WFPC2, but the spatial resolution is insufficient for performing photometry on the individual sources. All of these observations took place before 2017, but we can still investigate potential optical/infrared 


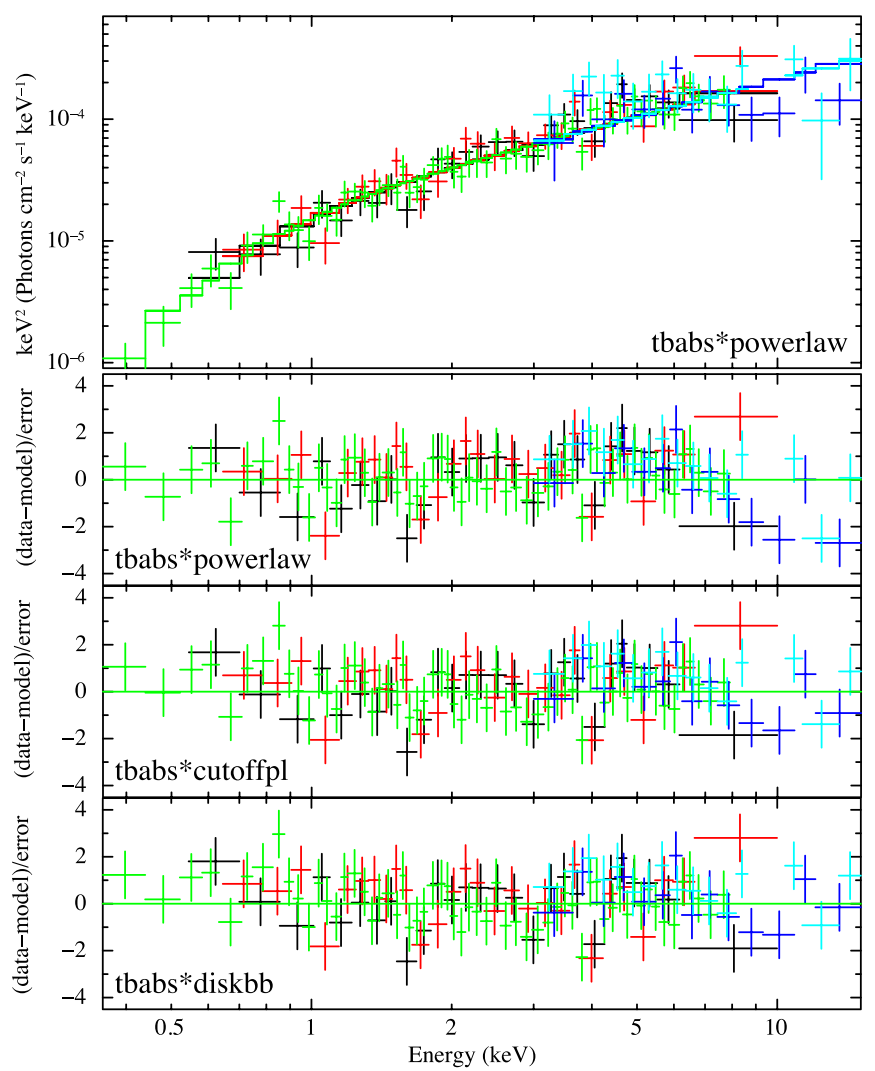

Figure 6. The XMM-Newton observation 0794581201 and NUSTAR observation 90302004004 joint spectrum of ULX-4, plotted with the best-fitting absorbed power-law model with $N_{\mathrm{H}}$ frozen to the Galactic value and $\Gamma=1.02 \pm 0.07$, along with residuals for a power-law and a cutoff powerlaw model.

Table 5

The ULX-4 Spectral-fitting Results for XMM-Newton Observation 0794581201 and NuSTAR Observation 90302004004

\begin{tabular}{|c|c|c|c|c|}
\hline $\begin{array}{l}\Gamma / T_{\text {in }} \\
(-/ \mathrm{keV})\end{array}$ & $\begin{array}{c}E_{\text {cut }} \\
(\mathrm{keV})\end{array}$ & \multicolumn{2}{|c|}{$\left(10^{-13} \mathrm{erg} \mathrm{cm}^{-2} \mathrm{~s}^{-1}\right)$} & $\chi^{2} /$ dof \\
\hline \multicolumn{5}{|c|}{ tbabs*powerlaw } \\
\hline $\begin{array}{r}1.00 \pm 0.05 \\
\text { tbabs } * \mathrm{cl}\end{array}$ & fpl & $3.2 \pm 0.1$ & $3.9 \pm 0.3$ & $174.2 / 143$ \\
\hline $0.7 \pm 0.1$ & $11_{-4}^{+9}$ & $3.2 \pm 0.1$ & $3.5 \pm 0.2$ & $158.4 / 142$ \\
\hline $\begin{array}{c}\text { tbabs*a } \\
4.3_{-0.5}^{+0.6}\end{array}$ & $\mathrm{ob}$ & $3.0 \pm 0.1$ & $3.2 \pm 0.1$ & $158.7 / 142$ \\
\hline
\end{tabular}

counterparts before the outburst. We corrected the astrometry (see Section 2) and derived a 0.' 9 error circle around the XMMNewton source position, which we found to be centered on a dark dust cloud. We find six potential counterparts around the edge of this error circle and a bright star cluster just outside it. We show the potential counterparts in Figure 11 in three of the HST bands and list them in Table 6-not all sources are visible in all bands. It is also feasible that the source is associated with the star cluster itself, although it is too crowded for us to characterize individual stars within it.

We used the DAOPHOT II/ALLSTAR software (Stetson 1987) to obtain photometric data for all six sources in the bands that they are detected in, and correct for Galactic reddening using $E(B-V)=0.2942 \pm 0.0028$ (Schlafly \& Finkbeiner 2011) and the Fitzpatrick (1999) reddening law. These sources are faint and in a crowded field, and given the presence of a dust lane, may be affected by significant local extinction. Therefore they are not detected at high significance, and these magnitudes should be considered approximations with large errors, especially at the lowest fluxes. We present a table of magnitudes in Table 6. Using a distance modulus of $\mu=29.16$ we convert these to absolute magnitudes. In addition, we use the $\mathrm{F} 606 \mathrm{~W}$ and $\mathrm{F} 814 \mathrm{~W}$ bands to produce a $V-I$ color.

\subsubsection{Foreground/Background Source}

ULX-4, if definitely located in its apparent host galaxy, is a highly unusual and interesting source. However, we must first assess whether it is a foreground or background contaminant.

Given NGC 6946's position relatively close to the Galactic plane, there is a higher density of foreground sources in its direction compared with galaxies at higher Galactic latitudes, therefore this is a possibility worth investigating. In addition, ULX-4 does not exhibit any significant absorption in addition to the Galactic column. This is unusual for ULXs, which tend to have local column densities $\sim 10^{21} \mathrm{~cm}^{-2}$ due to local absorption within their host galaxies (Winter et al. 2007).

If ULX-4 is a foreground source located within the Milky Way, it is very faint - at a distance of $10 \mathrm{kpc}$, its luminosity would be $3.4 \times 10^{33} \mathrm{erg} \mathrm{s}^{-1}$. Some Galactic sources such as V404 Cyg and Aql X-1 have quiescent luminosities similar to this (e.g., Garcia et al. 2001; Campana et al. 2014), but since ULX-4 has not previously been detected, implying a lower luminosity state with $L_{\mathrm{X}} \lesssim 10^{31} \mathrm{erg} \mathrm{s}^{-1}$ during the deepest of these observations, ULX-4 must be undergoing some form of outburst rather than being detected in a quiescent state. However, its detected luminosity is low for an outburst, even for very faint X-ray transients, which tend to have luminosities in the range of $10^{34}-10^{36} \mathrm{erg} \mathrm{s}^{-1}$ (e.g., Degenaar et al. 2012) during an outburst. Conversely, it is too high a luminosity for most cataclysmic variables aside from intermediate polars (IPs; e.g., Sazonov et al. 2006). IPs can possess hard spectra like that we find for ULX-4, but they are also expected to exhibit iron features (e.g., Kuulkers et al. 2006; Middleton et al. 2012), which we do not see in this source. In addition, the lack of an obvious foreground star detection limits any possible Galactic optical counterpart to dwarf stars. While we cannot entirely rule out ULX-4 being a foreground source on this basis, it would be an unusual source if it were.

ULX-4's coincidence with a dense, dusty region of one of NGC 6946's spiral arms, and lack of X-ray absorption beyond Galactic levels, suggests that it is likely not a background source either. Its outburst timescale is too short for a standard TDE (Komossa 2015) and too long for an X-ray flash or fast $\mathrm{X}$-ray transient resulting from an off-axis gamma-ray burst (e.g., Heise \& in 't Zand 2001; Yamazaki et al. 2002), and too hard a spectrum for either case.

Therefore we can be reasonably confident that ULX-4 is located within NGC 6946 rather than being a foreground or background source coincident with the galaxy.

\subsubsection{Supernova}

With its sudden appearance and increase in X-ray luminosity, we must also consider whether this source is an explosive transient. X-ray emission from core-collapse supernovae can have a range of luminosities, and the luminosity we observe for ULX-4 is a reasonable value for a supernova. However, the 

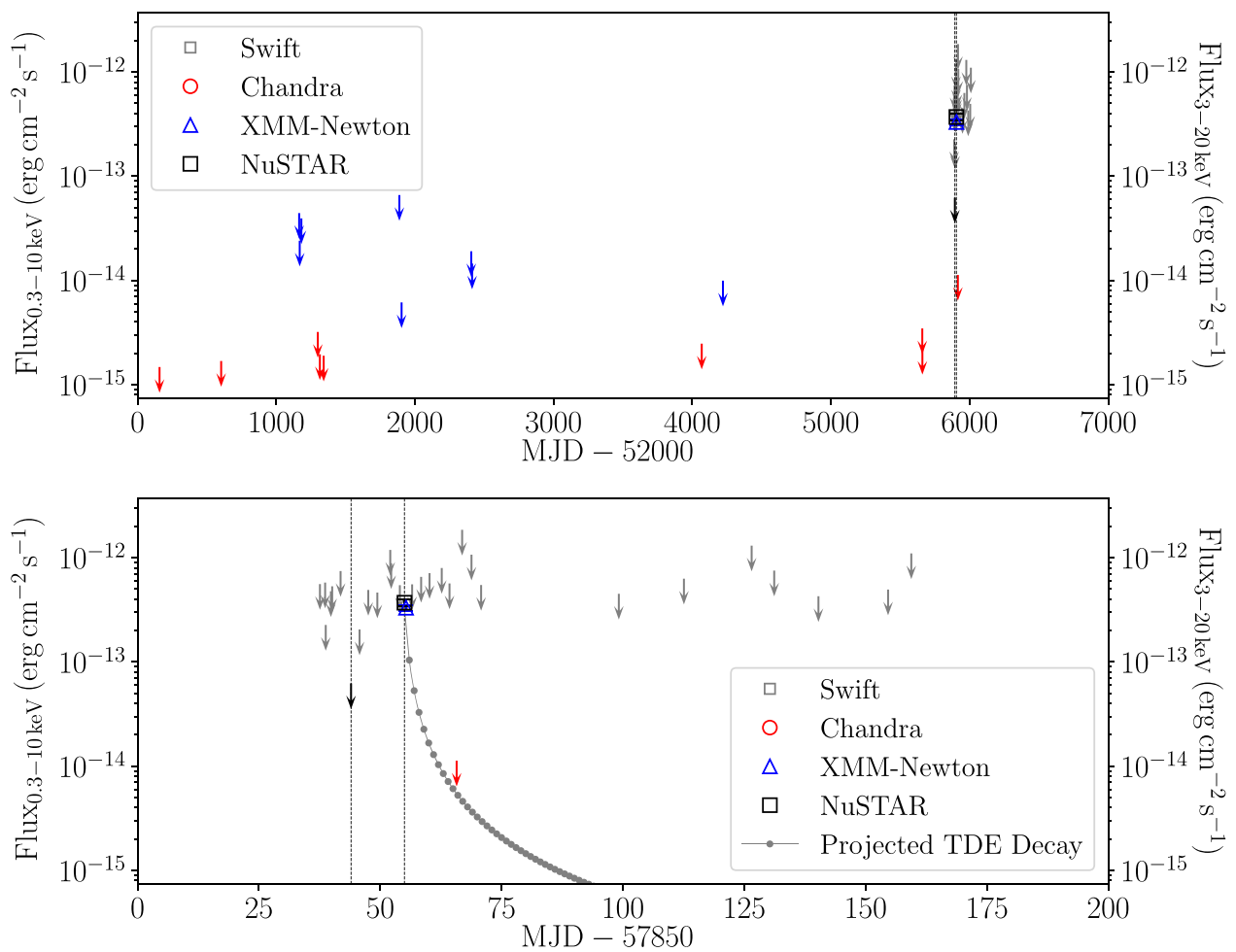

Figure 7. Long-term light curves for ULX-4, for the past $\sim 17 \mathrm{yr}$ (top) and for the observations in 2017 (bottom). The left axis denotes soft (0.3-10 keV) X-ray flux and applies to XMM-Newton (blue), Chandra (red), and Swift (gray) data points. The right axis denotes hard (3-20 keV) X-ray flux and applies to NuSTAR (black) data points. Detections are marked with symbols and upper limits by downward-pointing arrows. The times of the two NuSTAR observations are indicated by vertical black dashed lines. A $t^{-5 / 3}$ decay curve typical for tidal disruption events is projected from the XMM-Newton flux in gray circles.

XMM-Newton count rates (ct/s)

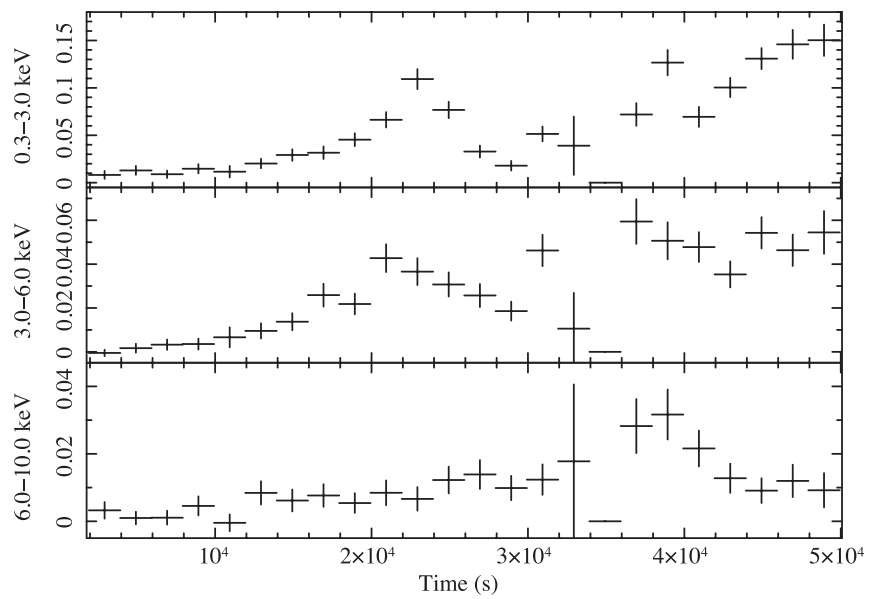

Figure 8. The XMM-Newton observation 0794581201 light curves for ULX-4 in the energy bands $0.3-3.0,3.0-6.0$, and $6.0-10.0 \mathrm{keV}$ energy bands, in $2000 \mathrm{~s}$ time bins. The dropout at $3.4 \times 10^{4} \mathrm{~s}$ corresponds to a period of soft proton flaring which was removed from the analysis and is not due to source behavior.

speed and extent of its subsequent decline in luminosity is far faster than expected for most supernovae (e.g., Dwarkadas \& Gruszko 2012), and there is no optical counterpart to the X-ray transient, nor a known optical transient at this position in the days before it appeared. Additionally, the short-timescale variability we observe supports the interpretation of ULX-4 being an accretion-powered object. Therefore we consider accretion-related explanations for this source.
XMM-Newton and NuSTAR count rates $(\mathrm{ct} / \mathrm{s})$

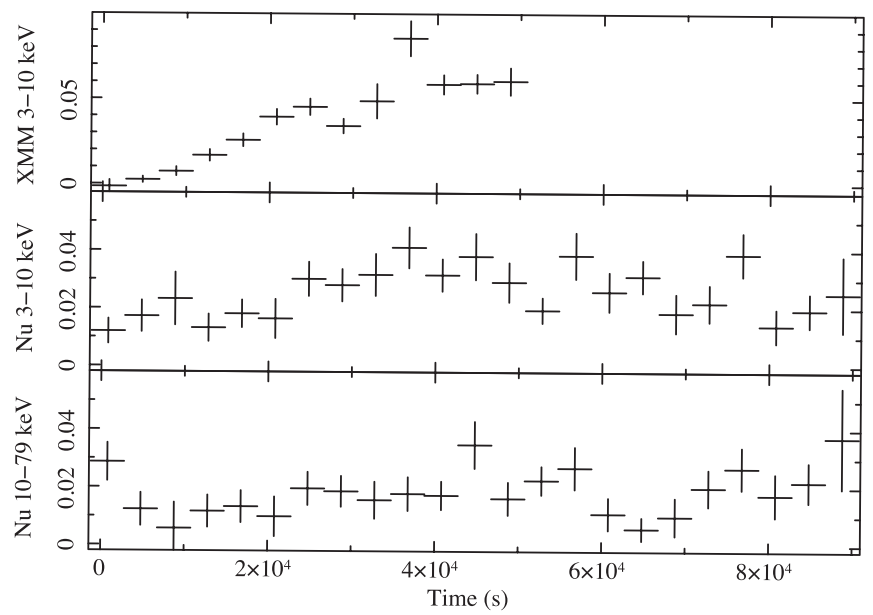

Figure 9. The XMM-Newton observation 0794581201 light curve for ULX-4 in the 3-10 keV energy band, along with the NUSTAR light curves in the 3-10 and $10-79 \mathrm{keV}$ energy bands, in $4000 \mathrm{~s}$ time bins.

\subsubsection{Super-Eddington Accreting Source}

It has become generally accepted that the majority of ULXs are stellar-mass compact objects accreting in a super-Eddington regime, and since ULX-4 reaches luminosities above $10^{39} \mathrm{erg} \mathrm{s}^{-1}$, this is a natural option to consider. Its hard spectrum, with evidence of a turnover at $\sim 10 \mathrm{keV}$, shows similarities with other ULXs in a hard ultraluminous regime (e.g., Sutton et al. 2013a; Walton et al. 2018a), some of which are confirmed NS ULXs. 


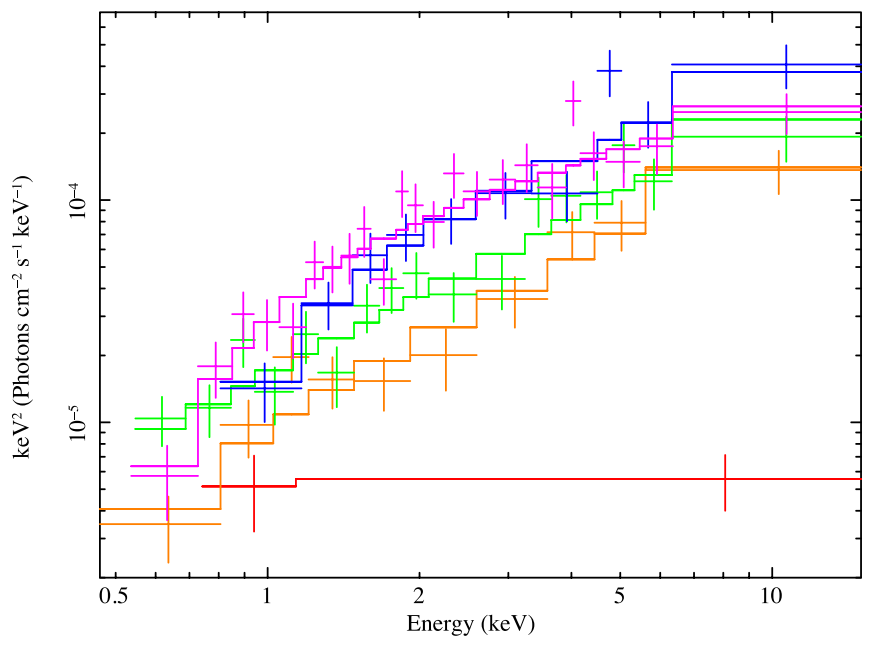

Figure 10. Time-resolved spectrum of ULX-4 from XMM-Newton observation 0794581201, divided into five $10 \mathrm{ks}$ intervals, in chronological order of red, orange, green, blue, and magenta. For clarity, only EPIC-pn data is shown.

If ULX-4 is such a source, this serendipitous detection offers unprecedented insight into the onset of super-Eddington accretion, as the source moves from a sub-Eddington to a super-Eddington state over the course of the observation. We find that, aside from a brief soft flare at early times, the onset of accretion is entirely dominated by hard emission, with no evidence of a significant disk component - though the signal quality may not be sufficient to detect the presence of a relatively dim disk. The spectrum as a whole only begins to show signs of softening at the end of the XMM-Newton observation, when the source is firmly in the ULX luminosity regime. This might suggest that the formation of a hot inner disk or accretion column precedes the buildup of an outer disk in this case - and with the timescale of the event being so short, it is possible that there was insufficient material available to form any kind of large disk before all matter was accreted. This is in contrast with the broadened-disk state seen in much of the lower luminosity ULX population and suggested to be objects accreting at $\sim$ Eddington rates, possibly in the process of transitioning into a super-Eddington ultraluminous state (e.g., Sutton et al. 2013b).

One other factor to consider is the transient nature of this source, being active for only $\sim 10$ days, assuming from the rise in luminosity over the observation that we are observing the source as it first appears. While many are highly variable, the majority of ULXs are reasonably persistent sources. One exception to this is the class of confirmed NS ULXs, most of which demonstrate periods of dramatic decrease in flux of well over an order of magnitude (e.g., Walton et al. 2015a; Fürst et al. 2016; Israel et al. 2017b), potentially due to the source entering the propeller regime. With the small sample size of sources demonstrating such behavior, the duty cycle of accretion and propeller state in these sources is not well understood. It is feasible that we are instead observing an NS briefly leaving and then returning to a propeller state, allowing only a brief onset of accretion. Since there are no previous observations of this source, it may be the case that we have witnessed its first onset of accretion, having been initially produced with a sufficiently high magnetic field and/or rotation frequency to begin in the propeller regime. However, our previous coverage of the source is not sufficient to rule out previous short outbursts in the past if the source simply has a low duty cycle.

All the potential optical counterparts we have identified in the HST data are consistent in brightness and color with being bright blue main sequence or OB-supergiant stars - as they are potentially affected by local extinction due to the presence of a dust lane, the latter case is more likely-except for source 4, and potentially 1 and 2, which have properties more consistent with red supergiants. Bright optical counterparts with $-8<M_{V}<-4$ are not uncommon for ULXs, though these counterparts may have significant contribution to their optical emission from the irradiated accretion disk rather than the companion star (e.g., Grisé et al. 2012; Fabrika et al. 2015; Ambrosi \& Zampieri 2018). We have no evidence that ULX-4 was accreting at the dates of the HST observations (although none of the observations were simultaneous with an X-ray observation so neither can we definitively rule this out). Under the assumption that ULX-4 is a new appearance, a blue accretion disk-dominated optical companion is less likely in this case, but this source may well be a high-mass X-ray binary with an optical counterpart dominated by the companion star. Red supergiants are also viable ULX counterparts (e.g., Heida et al. 2014), so none of the potential counterparts can be ruled out on that basis. It is possible that future HST observations will reveal changes in the optical/NIR emission that may aid in identifying a single counterpart.

An NS ULX briefly leaving the propeller regime is a reasonable explanation for this transient source. However, there are no pulsations detected from ULX-4 and no other definitive evidence that it is an NS. Therefore, we also explore other scenarios that could explain our results.

\subsubsection{Transient Outbursts}

Some transient ULXs are thought to be classical X-ray binary outbursts that happen to be luminous enough to briefly reach the ULX regime before declining in flux again (e.g., Middleton et al. 2013). While these sources usually undergo a transition from a low/hard to a high/soft state over the course of the outburst, the recent WATCHDOG survey (Tetarenko et al. 2016, and references therein) suggests that up to $40 \%$ of outbursts in Galactic BH binaries do not reach the soft state, with their spectra remaining hard over the duration of the outburst. Such hard transients do not always follow the fast-rise exponential-decay pattern seen in the more typical "canonical" outbursts (e.g., Brocksopp et al. 2004), and have timescales from the tens to hundreds of days.

However, there are several problems with a fast "hard-only" outburst scenario for ULX-4. First, the spectrum of ULX-4 is unusually hard even for such outbursting sources, which tend to have photon indices typical for the sub-Eddington hard state of $\Gamma=1.4-1.7$ (e.g., Revnivtsev et al. 2000; Belloni et al. 2002; Sidoli et al. 2011). Also, hard-only outbursts do not tend to reach the fluxes that full outbursts do, generally only reaching Eddington fractions of $\sim 10 \%$ (Tetarenko et al. 2016). Such an outburst would require ULX-4 to be an IMBH with mass $\sim 200 M_{\odot}$. It is possible for transient BHs in the hard state to reach up to $100 \%$ of Eddington before a transition to the soft thermal state (Dunn et al. 2010), though there is no evidence for such a transition taking place and it is unlikely that such an outburst could have concluded by the time of the latest Chandra non-detection of ULX-4. Therefore we conclude that 

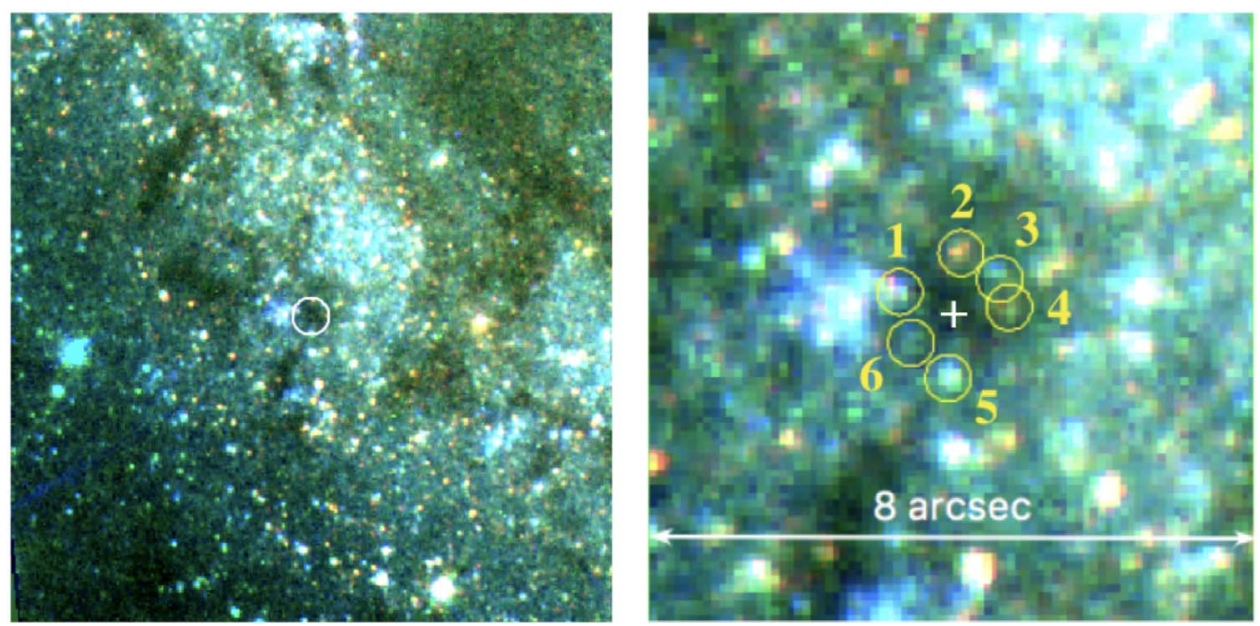

Figure 11. False-color HST images of the region around NGC 6946 ULX-4, with the near-infrared F110W band in red, F814W band in green, and F606W band in blue. Left, a $30 \times 30^{\prime}$ square around the source position, marked with a white $0 ! 990 \%$ position error circle. Left, a closer zoom in on the source region, with six potential optical/infrared counterparts labeled as in Table 6. We note that the IR data is not simultaneous with the optical data.

this kind of hard-only outburst is unlikely to be the cause of our detection of ULX-4.

The known source that may be a potential analog to ULX-4 is V404 Cyg, a low-mass X-ray binary that went into an outburst lasting tens of days in 2015 after being in a quiescent state for 26 yr. Instead of exhibiting the sub-Eddington states expected during most X-ray binary outbursts, it appeared to enter a super-Eddington regime, with a highly variable and often very hard spectrum, especially at its highest fluxes (Motta et al. 2017a). While the average luminosity over the course of the entire outburst was not super-Eddington, reaching $L_{\mathrm{X}}>10^{39} \mathrm{erg} \mathrm{s}^{-1}$ only during brief peaks in emission, much of its strong variability in flux and spectral shape can be attributed to varying levels of absorption of a slim disk inner accretion flow (e.g., Sánchez-Fernández et al. 2017), suggesting that its intrinsic luminosity may have been more consistently high. It does differ from ULX-4 in its strong emission in the International Gamma-Ray Astrophysics Laboratory band, with a reflection spectrum up to $100 \mathrm{keV}$ (Motta et al. 2017b) rather than the lower-energy turnover seen in ULXs. Nevertheless, the precedent for relatively shortduration super-Eddington outbursts accompanied by long periods of quiescence exists, and this may be a plausible explanation for ULX-4's behavior.

\subsubsection{Micro-tidal Disruption Events ( $\mu T D E s)$}

We have only observed ULX-4 once, so we cannot rule out a one-time transient scenario without a second detection of the source. A tidal disruption event (TDE) is one such scenario, and occurs when a star is disrupted and accreted onto a supermassive BH. Most TDEs have a spectrum that peaks in the ultraviolet or soft X-rays, and decline on a timescale of months to years with a characteristic $t^{-5 / 3}$ power-law drop-off in flux due to the rate of matter fallback (for a recent review of TDE observations, see Komossa 2015).

As this source is not at the center of its host galaxy and possesses a hard spectrum rather than a soft one, a typical TDE does not appear to be a good match for our observations of ULX-4. However, it has been suggested that $\mu$ TDEs, in which a low-mass star or large planet is disrupted or partially disrupted by a stellar-mass BH or IMBH, may also occur and may possess different observational signatures than typical TDEs (e.g., Perets et al. 2016).

If we assume that ULX-4 reaches its peak luminosity during the joint XMM-Newton and NUSTAR observation and decays according to a typical $t^{-5 / 3}$ power law immediately afterwards, we find that the Chandra non-detection upper limit is actually consistent with such a scenario (see Figure 7). Even if there is a delay before the source flux begins to decay, some simulations indicate that a small $\mathrm{BH}$ mass or an ultra-close encounter with an IMBH could lead to a high fallback rate, early intersection of disrupted matter, and very rapid accretion disk formation in close proximity to the $\mathrm{BH}$ (possibly subject to general relativistic effects), instead of the $t^{-5 / 3}$ decline from typical self-interaction of the disrupted matter (e.g., Evans et al. 2015; Kawana et al. 2018). The rapid accumulation of an accretion disk would lead to a brief period of super-Eddington accretion in which the disk matter is drained, with a timescale of $10^{5}-10^{6}$ s (e.g., Perets et al. 2016). This is consistent with our observation of ULX-4.

Some fast and bright X-ray transients have previously been attributed to the potential tidal disruption of a star by an IMBH (e.g., Jonker et al. 2013, though this example is still brighter and softer than ULX-4). We can also compare ULX-4 to wellstudied central TDEs such as the J1644+57 event in 2011 (Burrows et al. 2011; Levan et al. 2011), which is particularly relevant as a "jetted TDE" with properties different from more typical TDEs - namely a hard spectrum and a super-Eddington luminosity (a second source, J2058+05, has similar properties and is also thought to be a jetted TDE; Cenko et al. 2012). It has been argued that J1644+57 demonstrates the properties of a scaled-up, transient ULX (e.g., Socrates 2012; Kara et al. 2016), so it may be the case that the transient ULX-4 is a scaled-down example of a similar event. Its luminosity was far higher than ULX-4's, reaching $\sim 10^{48} \mathrm{erg} \mathrm{s}^{-1}$ at its brightest, and a naive scaling of mass with peak luminosity would suggest a compact object a fraction of the mass of the Sun for ULX-4, so there would have to be a substantial difference in Eddington ratio in play as well if these sources have a similar origin. In early times it demonstrated flaring behavior on $\sim$ hour timescales, to which the flare during ULX-4's increase in brightness could be analogous. ULX-4's spectrum is far harder even than that of $\mathrm{J} 1644+57$, which at $\Gamma \sim 2$ is already harder 
Table 6

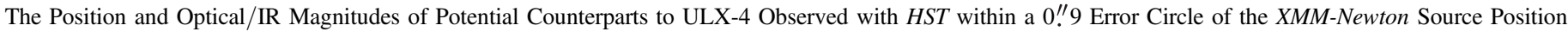

\begin{tabular}{|c|c|c|c|c|c|c|c|c|c|}
\hline ID & Position (J2000) & $\begin{array}{c}m_{547 \mathrm{M}} \\
\text { (2001 Apr 22) }\end{array}$ & $\begin{array}{l}m_{606 \mathrm{~W}} \\
\quad(2000\end{array}$ & $\begin{array}{l}m_{814 \mathrm{~W}} \\
\text { ec } 25)\end{array}$ & $\begin{array}{l}m_{110 \mathrm{~W}} \\
\quad(201\end{array}$ & $\begin{array}{l}m_{128 \mathrm{~N}} \\
\text { eb 9) }\end{array}$ & $M_{606}$ & $M_{814}$ & $V-I$ \\
\hline 1 & $20: 34: 56.99+60: 08: 13.37$ & $24.8 \pm 0.3$ & $23.0 \pm 0.6$ & $24.1 \pm 0.3$ & $20.6 \pm 0.2$ & $23.7 \pm 0.3$ & $-6.2 \pm 0.6$ & $-5.0 \pm 0.3$ & $-1.2 \pm 0.7$ \\
\hline 2 & $20: 34: 56.88+60: 08: 13.88$ & $>26.4$ & $25 \pm 2$ & $26 \pm 2$ & $20.9 \pm 0.1$ & $23.8 \pm 0.3$ & $-4 \pm 2$ & $-3 \pm 2$ & $-1 \pm 3$ \\
\hline 3 & $20: 34: 56.82+60: 08: 13.54$ & $24.9 \pm 0.5$ & $24.2 \pm 0.8$ & $25 \pm 1$ & $24 \pm 4$ & $26.3 \pm 1$ & $-4.9 \pm 0.8$ & $-4 \pm 1$ & $-1 \pm 1$ \\
\hline 4 & $20: 34: 56.80+60: 08: 13.17$ & $>26.4$ & $>26.7$ & $26 \pm 4$ & $22.0 \pm 0.1$ & $25.1 \pm 0.1$ & $>-2.4$ & $-3 \pm 4$ & $>0.6$ \\
\hline 5 & $20: 34: 56.90+60: 08: 12.25$ & $24.0 \pm 0.2$ & $23.0 \pm 0.5$ & $23.3 \pm 0.4$ & $21.5 \pm 0.1$ & $24.8 \pm 0.1$ & $-6.2 \pm 0.5$ & $-5.9 \pm 0.4$ & $-0.3 \pm 0.6$ \\
\hline 6 & $20: 34: 56.97+60: 08: 12.71$ & $26 \pm 1$ & $24.3 \pm 0.8$ & $26 \pm 2$ & $>28.3$ & $25.2 \pm 0.2$ & $-4.8 \pm 0.8$ & $-3 \pm 2$ & $-2 \pm 2$ \\
\hline
\end{tabular}

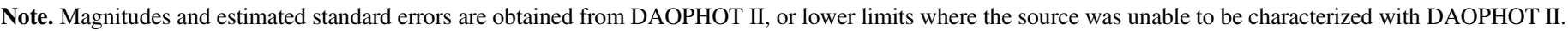
Absolute magnitudes are calculated assuming a distance modulus $\mu=29.16$. The date that each $H S T$ observation was taken is given in the header.

than all other TDEs observed to date (Auchettl et al. 2017). However, J1644 +57 does demonstrate a softening of its spectrum with its drop in flux, exhibiting a photon index closer to $\Gamma \sim 1.5$ at its highest fluxes at early times. Therefore it may be feasible for a $\mu \mathrm{TDE}$ to exhibit a very hard spectrum at its earliest times. Finally, J1644+57 as a jetted TDE is also distinct from other TDEs in that it has a very high X-ray to optical flux ratio, evidence of less reprocessing of X-ray emission into the optical regime. It also possesses the lowest column densities compared to other TDEs. The lack of a bright optical counterpart to ULX-4 and the absence of an absorption component beyond the Galactic contribution in ULX-4 bears some similarities to this scenario.

Should ULX-4 be a super-Eddington $\mu \mathrm{TDE}$, the observed luminosity would imply a BH mass of $\sim 10 M_{\odot}$, which is low to induce tidal disruption in most cases but may still do so for a low-mass object. Under the assumption that the highest luminosity we observe is the peak luminosity of a $\mu \mathrm{TDE}$, $L_{\text {peak }}=3.2 \times 10^{39} \mathrm{erg} \mathrm{s}^{-1}$, this is consistent with the luminosity expected from the tidal disruption of a $\sim 0.01 M_{\odot}$ brown dwarf by a $16 M_{\odot} \mathrm{BH}$.

Assuming a $t^{-5 / 3}$ decline from a maximum flux observed during the joint XMM-Newton and NUSTAR observation and no further cutoff, we project the flux at the time of writing this paper to be $<10^{-17} \mathrm{erg} \mathrm{s}^{-1}$, so unfortunately the opportunity to feasibly confirm such a decay law has long since passed. However with the data we currently possess, a $\mu \mathrm{TDE}$ analogous to the super-Eddington jetted TDE scenario, with a stellar-mass BH disrupting a brown dwarf and undergoing a subsequent brief period of super-Eddington accretion, is a plausible explanation for ULX-4.

\section{Conclusions}

NGC 6946 remains a valuable galaxy for the study of superEddington accretion. Both ULX-1 and ULX-2 possess very steep power-law spectra and are potential examples of ultraluminous supersoft sources dominated by outer disk and wind emission, even though their luminosity is usually in the Eddington threshold regime of $10^{38}-10^{39} \mathrm{erg} \mathrm{s}^{-1}$. In fact, NGC 6946 contains other sources within this luminosity regime, which may also offer examples of similar behavior and further opportunities to study the effects of superEddington accretion beyond the ULX population. While their lower luminosity makes them more challenging than ULXs to study, the reasonably low distance to NGC 6946 makes it possible to place decent constraints on the spectrum with moderate-to-long observations with XMM-Newton, and will make them a highly interesting population to examine with next-generation X-ray missions such as Athena (Nandra et al. 2013).

Given that ULX-3 was not the primary target, the 2017 NuSTAR observations were not optimized to characterize it. Even so, despite it being a reasonably soft ULX with $\Gamma=2.51 \pm 0.05, N u S T A R$ is able to detect it almost to $20 \mathrm{keV}$. We do not significantly detect a high-energy turnover as seen in the spectra of other ULXs, but due to the softness of the spectrum and the potential presence of a steep power law after such a break (as seen in the best quality NuSTAR data of hard ULXs), identifying such a break would be difficult. While NUSTAR is most suitable for studying ULXs with hard spectra, this does go to show that softer ULXs can also be detected and studied with this mission.

The serendipitous discovery of ULX-4 provides a fascinating potential example of the onset of super-Eddington accretion. Its very hard spectrum, equally consistent with a cutoff power-law model and a hot disk blackbody, and transient nature make it challenging to identify. However, it bears similarity to the observed hard spectra of NS ULXs, which also have the capacity to undergo sudden and dramatic rises and falls in flux due to the propeller effect, although we are unable to detect pulsations from ULX-4 (and would not necessarily expect to given the number of photons observed from the source). Additionally, the brief duration of its appearance and lack of previous detections raise questions about whether the object has a particularly low duty cycle or whether it is a new system. Alternatively, the outburst could be consistent with a superEddington outburst similar to V404 Cyg, although it does not exhibit the same reflection-dominated spectrum at high energies, or a $\mu \mathrm{TDE}$ in which a low-mass object such as a brown dwarf is disrupted by a stellar-mass compact object, although its spectrum is harder even than the jetted TDEs previously observed. Ultimately, further detections of ULX-4 would be required to establish whether it is an NS ULX or other relatively persistently accreting object rather than a transient event.

We thank our anonymous referee for useful comments on this paper. This work was supported under NASA contract NNG08FD60C. D.J.W. acknowledges financial support from STFC in the form of an Ernest Rutherford fellowship. This work made use of data from the NUSTAR mission, a project led by the California Institute of Technology, managed by the Jet Propulsion Laboratory, and funded by the National Aeronautics and Space Administration. This work has also made use of observations by XMM-Newton, an ESA science mission with instruments and contributions directly funded by ESA Member States and NASA. Results reported in this article are based in 
part on public data obtained from the Chandra and Swift data archives, and on observations made with the NASA/ESA Hubble Space Telescope, obtained from the Data Archive at the Space Telescope Science Institute, which is operated by the Association of Universities for Research in Astronomy, Inc., under NASA contract NAS 5-26555.

Facilities: NuSTAR, XMM, CXO, Swift(XRT), HST.

Software: astropy (Astropy Collaboration et al. 2013, 2018), CIAO (Fruscione et al. 2006), HENDRICS (Bachetti 2015), HEASoft (NASA's High Energy Astrophysics Science Archive Research Center (HEASARC), 2014), NuSTARDAS, XMMNewton SAS.

\section{ORCID iDs}

Hannah P. Earnshaw (iD https://orcid.org/0000-00015857-5622

Brian W. Grefenstette (iD https://orcid.org/0000-00021984-2932

Dominic J. Walton (i) https://orcid.org/0000-0001-5819-3552

Didier Barret (1D https://orcid.org/0000-0002-0393-9190

Felix Fürst (iD https://orcid.org/0000-0003-0388-0560

Marianne Heida (10 https://orcid.org/0000-0002-1082-7496

Sean N. Pike (1) https://orcid.org/0000-0002-8403-0041

Daniel Stern (1D https://orcid.org/0000-0003-2686-9241

\section{References}

Abolmasov, P., Fabrika, S., Sholukhova, O., \& Kotani, T. 2008, arXiv:0809.0409

Ambrosi, E., \& Zampieri, L. 2018, MNRAS, 480, 4918

Anand, G. S., Rizzi, L., \& Tully, R. B. 2018, AJ, 156, 105

Arnaud, K. A. 1996, in ASP Conf. Ser. 101, Astronomical Data Analysis Software and Systems V, ed. G. H. Jacoby \& J. Barnes (San Francisco, CA: ASP), 17

Astropy Collaboration, Price-Whelan, A. M., Sipőcz, B. M., et al. 2018, AJ, 156,123

Astropy Collaboration, Robitaille, T. P., Tollerud, E. J., et al. 2013, A\&A, 558, A33

Auchettl, K., Guillochon, J., \& Ramirez-Ruiz, E. 2017, ApJ, 838, 149

Bachetti, M. 2015, MaLTPyNT: Quick look timing analysis for NuSTAR data, Astrophysics Source Code Library, ascl:1502.021

Bachetti, M., Harrison, F. A., Walton, D. J., et al. 2014, Natur, 514, 202

Bachetti, M., Rana, V., Walton, D. J., et al. 2013, ApJ, 778, 163

Bauer, F. E., \& Brandt, W. N. 2004, ApJL, 601, L67

Belloni, T., Colombo, A. P., Homan, J., Campana, S., \& van der Klis, M. 2002, A\&A, 390, 199

Berghea, C. T., \& Dudik, R. P. 2012, ApJ, 751, 104

Berghea, C. T., Dudik, R. P., Tincher, J., \& Winter, L. M. 2013, ApJ, 776, 100

Brightman, M., Harrison, F., Walton, D. J., et al. 2016, ApJ, 816, 60

Brightman, M., Harrison, F. A., Fürst, F., et al. 2018, NatAs, 2, 312

Brocksopp, C., Bandyopadhyay, R. M., \& Fender, R. P. 2004, NewA, 9, 249

Burrows, D. N., Kennea, J. A., Ghisellini, G., et al. 2011, Natur, 476, 421

Campana, S., Brivio, F., Degenaar, N., et al. 2014, MNRAS, 441, 1984

Carpano, S., Haberl, F., Maitra, C., \& Vasilopoulos, G. 2018, MNRAS, 476, L45

Carpano, S., Pollock, A. M. T., Wilms, J., Ehle, M., \& Schirmer, M. 2007, A\&A, 461, L9

Cenko, S. B., Krimm, H. A., Horesh, A., et al. 2012, ApJ, 753, 77

Colbert, E. J. M., \& Mushotzky, R. F. 1999, ApJ, 519, 89

Degenaar, N., Wijnands, R., Cackett, E. M., et al. 2012, A\&A, 545, A49

Devi, A. S., Misra, R., Shanthi, K., \& Singh, K. Y. 2008, ApJ, 682, 218

Di Stefano, R., Kong, A. K. H., Greiner, J., et al. 2004, ApJ, 610, 247

Dunn, R. J. H., Fender, R. P., Körding, E. G., Belloni, T., \& Cabanac, C. 2010, MNRAS, 403, 61

Dwarkadas, V. V., \& Gruszko, J. 2012, MNRAS, 419, 1515

Earnshaw, H. M., \& Roberts, T. P. 2017, MNRAS, 467, 2690

Earnshaw, H. P., Roberts, T. P., \& Sathyaprakash, R. 2018, MNRAS, 476, 4272

Evans, C., Laguna, P., \& Eracleous, M. 2015, ApJL, 805, L19

Fabbiano, G., \& Trinchieri, G. 1987, ApJ, 315, 46
Fabrika, S., Ueda, Y., Vinokurov, A., Sholukhova, O., \& Shidatsu, M. 2015, NatPh, 11, 551

Fitzpatrick, E. L. 1999, PASP, 111, 63

Fridriksson, J. K., Homan, J., Lewin, W. H. G., Kong, A. K. H., \& Pooley, D. 2008, ApJS, 177, 465

Fruscione, A., McDowell, J. C., Allen, G. E., et al. 2006, Proc. SPIE, 6270, $62701 \mathrm{~V}$

Fürst, F., Walton, D. J., Harrison, F. A., et al. 2016, ApJL, 831, L14

Garcia, M. R., McClintock, J. E., Narayan, R., et al. 2001, ApJL, 553, L47

Gladstone, J. C., Copperwheat, C., Heinke, C. O., et al. 2013, ApJS, 206, 14

Gladstone, J. C., Roberts, T. P., \& Done, C. 2009, MNRAS, 397, 1836

Grisé, F., Kaaret, P., Corbel, S., et al. 2012, ApJ, 745, 123

Grisé, F., Kaaret, P., Corbel, S., Cseh, D., \& Feng, H. 2013, MNRAS, 433, 1023

Heida, M., Jonker, P. G., Torres, M. A. P., et al. 2014, MNRAS, 442, 1054

Heise, J., \& in 't Zand, J 2001, arXiv:astro-ph/0112353

Holt, S. S., Schlegel, E. M., Hwang, U., \& Petre, R. 2003, ApJ, 588, 792

Huppenkothen, D., Bachetti, M., Stevens, A. L., et al. 2019, arXiv:1901.07681

Illarionov, A. F., \& Sunyaev, R. A. 1975, A\&A, 39, 185

Israel, G. L., Belfiore, A., Stella, L., et al. 2017a, Sci, 355, 817

Israel, G. L., Papitto, A., Esposito, P., et al. 2017b, MNRAS, 466, L48

Jonker, P. G., Glennie, A., Heida, M., et al. 2013, ApJ, 779, 14

Kaaret, P., Feng, H., \& Roberts, T. P. 2017, ARA\&A, 55, 303

Kaaret, P., Feng, H., Wong, D. S., \& Tao, L. 2010, ApJL, 714, L167

Kara, E., Miller, J. M., Reynolds, C., \& Dai, L. 2016, Natur, 535, 388

Kawana, K., Tanikawa, A., \& Yoshida, N. 2018, MNRAS, 477, 3449

Koliopanos, F., Vasilopoulos, G., Godet, O., et al. 2017, A\&A, 608, A47

Komossa, S. 2015, JHEAp, 7, 148

Kuulkers, E., Norton, A., Schwope, A., \& Warner, B. 2006, in Compact Stellar X-ray Sources, ed. W. Lewin \& M. van der Klis (Cambridge: Cambridge Univ. Press), 421

Levan, A. J., Tanvir, N. R., Cenko, S. B., et al. 2011, Sci, 333, 199

Lin, D., Irwin, J. A., Webb, N. A., Barret, D., \& Remillard, R. A. 2013, ApJ, 779,149

Liu, J.-F., \& Bregman, J. N. 2005, ApJS, 157, 59

Matonick, D. M., \& Fesen, R. A. 1997, ApJS, 112, 49

Middleton, M. J., Cackett, E. M., Shaw, C., et al. 2012, MNRAS, 419, 336

Middleton, M. J., Heil, L., Pintore, F., Walton, D. J., \& Roberts, T. P. 2015a, MNRAS, 447, 3243

Middleton, M. J., Miller-Jones, J. C. A., Markoff, S., et al. 2013, Natur, 493, 187

Middleton, M. J., Walton, D. J., Fabian, A., et al. 2015b, MNRAS, 454, 3134

Motta, S. E., Kajava, J. J. E., Sánchez-Fernández, C., et al. 2017a, MNRAS, 471, 1797

Motta, S. E., Kajava, J. J. E., Sánchez-Fernández, C., Giustini, M., \& Kuulkers, E. 2017b, MNRAS, 468, 981

Mukherjee, E. S., Walton, D. J., Bachetti, M., et al. 2015, ApJ, 808, 64

Nandra, K., Barret, D., Barcons, X., et al. 2013, arXiv:1306.2307

NASA's High Energy Astrophysics Science Archive Research Center (HEASARC) 2014, HEAsoft: Unified Release of FTOOLS and XANADU, Astrophysics Source Code Library, ascl:1408.004

Perets, H. B., Li, Z., Lombardi, J. C., Jr., \& Milcarek, S. R., Jr. 2016, ApJ, 823,113

Pinto, C., Middleton, M. J., \& Fabian, A. C. 2016, Natur, 533, 64

Pintore, F., Belfiore, A., Novara, G., et al. 2018, MNRAS, 477, L90

Pintore, F., Zampieri, L., Stella, L., et al. 2017, ApJ, 836, 113

Rao, F., Feng, H., \& Kaaret, P. 2010, ApJ, 722, 620

Revnivtsev, M. G., Borozdin, K. N., Priedhorsky, W. C., \& Vikhlinin, A. 2000, ApJ, 530, 955

Roberts, T. P., \& Colbert, E. J. M. 2003, MNRAS, 341, L49

Rodríguez Castillo, G. A., Israel, G. L., Belfiore, A., et al. 2019, ApJ, submitted (arXiv:1906.04791)

Sánchez-Fernández, C., Kajava, J. J. E., Motta, S. E., \& Kuulkers, E. 2017, A\&A, 602, A40

Sathyaprakash, R., Roberts, T. P., Walton, D. J., et al. 2019, MNRAS, 488, L35

Sazonov, S., Revnivtsev, M., Gilfanov, M., Churazov, E., \& Sunyaev, R. 2006, A\&A, 450, 117

Schlafly, E. F., \& Finkbeiner, D. P. 2011, ApJ, 737, 103

Sidoli, L., Paizis, A., Mereghetti, S., Götz, D., \& Del Santo, M. 2011, MNRAS, 415, 2373

Socrates, A. 2012, ApJL, 756, L1

Soria, R., Kuntz, K. D., Winkler, P. F., et al. 2012, ApJ, 750, 152

Stella, L., White, N. E., \& Rosner, R. 1986, ApJ, 308, 669

Stetson, P. B. 1987, PASP, 99, 191

Stobbart, A.-M., Roberts, T. P., \& Wilms, J. 2006, MNRAS, 368, 397 
Sutton, A. D., Roberts, T. P., Gladstone, J. C., et al. 2013a, MNRAS, 434, 1702

Sutton, A. D., Roberts, T. P., \& Middleton, M. J. 2013b, MNRAS, 435, 1758 Sutton, A. D., Roberts, T. P., Walton, D. J., Gladstone, J. C., \& Scott, A. E. 2012, MNRAS, 423, 1154

Tetarenko, B. E., Sivakoff, G. R., Heinke, C. O., \& Gladstone, J. C. 2016, ApJS, 222, 15

Tsygankov, S. S., Mushtukov, A. A., Suleimanov, V. F., \& Poutanen, J. 2016, MNRAS, 457, 1101
Urquhart, R., \& Soria, R. 2016, MNRAS, 456, 1859

Walton, D. J., Fürst, F., Harrison, F. A., et al. 2018a, MNRAS, 473, 4360

Walton, D. J., Fürst, F., Heida, M., et al. 2018b, ApJ, 856, 128

Walton, D. J., Harrison, F. A., Bachetti, M., et al. 2015a, ApJ, 799, 122

Walton, D. J., Harrison, F. A., Grefenstette, B. W., et al. 2014, ApJ, 793, 21

Walton, D. J., Middleton, M. J., Rana, V., et al. 2015b, ApJ, 806, 65

Wilms, J., Allen, A., \& McCray, R. 2000, ApJ, 542, 914

Winter, L. M., Mushotzky, R. F., \& Reynolds, C. S. 2007, ApJ, 655, 163

Yamazaki, R., Ioka, K., \& Nakamura, T. 2002, ApJL, 571, L31 